\title{
Numerical study on toroidal mode coupling and triggering of neoclassical tearing modes by sawteeth
}

\author{
Q. Yu, S. Günter, K. Lackner, E. Strumberger, and V. Igochine \\ Max-Planck-Institut für Plasmaphysik, 85748 Garching, Germany
}

\begin{abstract}
Numerical simulations have been carried out to understand the seeding of neoclassical tearing modes (NTMs), using a single fluid model and the four-field equations. The paper starts with considering a simplified physics situation: the destabilization of a linearly stable $m / n=3 / 2$ mode by an unstable $m / n=2 / 2$ mode ( $\mathrm{m} / \mathrm{n}$ being the poloidal/toroidal mode number). Within single fluid model the mode coupling is found to be weakened by relative mode rotation and enhanced by finite plasma $\beta$ value. For the four-field equations in case of a sufficiently low electron diamagnetic drift frequency, the results are similar to those from the single fluid equations. For a sufficiently large diamagnetic drift frequency, three regimes are found: (a) Suppression regime for moderate plasma $\beta$ values, in which the originally unstable $2 / 2$ mode is stabilized by its coupling to the originally stable 3/2 mode; (b) Oscillation regime for a sufficiently large $\beta$ value, in which the 3/2 mode has the feature of an ideal mode, and the diamagnetic drift frequency reaches the shear Alfven frequency outside the tearing layer; (c) NTM regime for sufficiently large $\beta$ value and bootstrap current density. Consistent with these findings, in our simulations 3/2 modes are triggered by sufficiently strong sawtooth crashes at high $\beta$ value and/or a low electron diamagnetic drift frequency. A sufficiently large bootstrap current density is then required for the 3/2 island to grow into the NTM regime.
\end{abstract}




\section{Introduction}

It is well known that toroidal geometry results in coupling of modes with different poloidal mode numbers $m$, in particular to modes with mode numbers $(m+1)$ and $(m-1)$ at same toroidal mode numbers $n$. Experimental results have shown the importance of such mode coupling. For example, sawtooth crashes can trigger neoclassical tearing modes (NTMs) for sufficiently high plasma $\beta$ values in tokamak experiments, which usually degrade plasma confinement or even lead to plasma disruption [1-11]. The large $m / n=1 / 1$ mode during the sawtooth crashes can possibly trigger the $m / n=2 / 1$ mode via toroidal mode coupling. However, in most experiments the nonlinear harmonic of the $1 / 1$ mode, the 2/2 component, is found to drive the 3/2 NTM more frequently [1-9]. Due to the importance of NTMs for tokamak power plants, the understanding of the physics involved in the triggering of NTMs is a very important issue. During major disruptions in tokamak plasmas, magnetic islands of different helicities or even stochastic field can be generated via mode coupling, usually in addition to a large 2/1 mode, allowing the plasma energy to be released in a short time scale [12-13]. Due to toroidal mode coupling, edge localized modes (ELMs) might also drive islands of different helicities and cause local stochastic field in the edge region of $\mathrm{H}$-mode plasmas, resulting in a release of $\sim 10 \%$ of the total plasma energy within a short time scale ( 1ms) [e.g. 14].

Since 1970s, extensive theoretical efforts have been devoted to understand the stability properties in toroidal geometry, including both analytical and numerical studies [15-34]. Without differential plasma rotation, a mode with one $n$ number might have several large $m$ components, in particular in strongly shaped plasmas [32]. Thus, an unstable tearing mode, e.g., the $m / n=2 / 1$ mode, will also lead to other islands, such as the 3/1 and 4/1 islands, in toroidal geometry. Differential plasma rotation, however, can weaken the mode coupling, as shown in analytical theory [16-18].

Analytical theories reveal that without mode coupling, the stability of a single magnetic island is determined by the tearing mode stability index $\Delta^{\prime}$, bootstrap current density, Glasser effect, the 
diamagnetic drift, and the associated ion polarization current [35-40]. Among them, the diamagnetic drift is quite important for the stability of a small island. Linear tearing modes are predicted to be stable in high temperature plasmas due to electron diamagnetic drift even for a positive $\Delta^{\prime}$ [41], as also seen from two-fluid numerical studies in cylinder geometry $[42,43]$.

As one of the major triggers for the NTM onset and generally as a typical phenomenon in tokamak experiments, sawteeth have attracted much research interest. Simulations in toroidal geometry have demonstrated that diamagnetic stabilization is essential to explain the quasi-periodic sawtooth cycles [44-46]. The very fast dynamics of a single sawtooth crash has been explained by the effect of the parallel electron pressure gradient in Ohm's law. Fast sawtooth crashes and associated strong parallel electrical fields have been derived from two-fluid simulations for high temperature plasmas (2keV) in cylinder geometry $[47,48]$. Detailed investigations of the reconnection region during a sawtooth crash also indicate of the importance of two-fluid physics in toroidal geometry simulations [49].

The triggering of NTMs by sawteeth have been simulated before based on single fluid equations [50,51], taking into account the time evolution of $\Delta^{\prime}$ and bootstrap current perturbation, but neglecting the differential plasma rotation in the nonlinear calculations [50]. In Ref. [51] the ion polarization current was included, but assumed to be proportional to the bootstrap current perturbation and inversely proportional to the magnetic island width, while two-fluid physics as well as the differential plasma rotation were neglected. In these simulation a destabilization of the $m / n=3 / 2$ mode by the internal kink mode has been found $[50,51]$. Linear studies in toroidal geometry have revealed the importance of toroidal mode coupling as well as the diamagnetic drift for mode growth [52]. The sawtooth crash is found to lead to an increase in the $\Delta^{\prime}$ value of the driven mode [53].

For the case that the magnetic shear is sufficiently small over a large region in the core, numerical studies have shown fast growing infernal modes coupled to poloidal tearing sideband, driving magnetic islands on neighboring rational surfaces [54,55]. In these simulations the bootstrap current drive as well 
as the stabilizing Glasser effect are considered. Any shielding effects due to differential diamagnetic drift or plasma rotation have however been neglected [54,55].

Due to the complex nonlinearity and mode coupling in toroidal geometry, numerical calculations of nonlinear MHD instabilities are extremely challenging and have to be limited to certain physics issues. The input parameters for calculations, such as the Lundquist number (the ratio between the resistive time and the Alfven time), are usually well below experimental ones.

In this paper, the toroidal mode coupling and the triggering of NTMs by sawteeth are studied numerically, based on the four-field equations [26]. The large aspect ratio expansion has been applied for obtaining these equations, and higher order terms, such as the magnetic field curvature are not accurately described [26]. Therefore, the approximations associated with the four-field model are particularly severe for the linear $\mathrm{m} / \mathrm{n}=1 / 1$ mode, which is always unstable in our model if the $\mathrm{q}$ value drops well below 1 for a relatively low diamagnetic drift frequency. When the 1/1 mode amplitude is sufficiently large such that the plasma pressure is flattened across the $1 / 1$ island, the curvature effect is however expected to be less important. The main focus of this paper is not on the stability of the $1 / 1$ mode itself, but rather on its effect on neighboring resonant surfaces when its amplitude is sufficiently large. For such studies it is important that these equations include toroidal mode coupling, diamagnetic drift and the associated ion polarization current, bootstrap current, and differential plasma rotation, the key physics elements for the NTM onset as expected from analytical theories [5,35-40].

As the equations of this model are relatively simple compared to the full MHD equations, numerical calculations are less time-consuming and thus allow the use of realistic experimental parameters as input. Our calculations are limited to the triggering of $m / n=3 / 2$ modes by $2 / 2$ perturbations. The effects of the plasma $\beta$ value, bootstrap current density, electron diamagnetic drift, and differential plasma rotation on mode coupling are studied. It will be shown that the two-fluid physics such as diamagnetic drift, neglected in previous nonlinear studies [50,51], is important for toroidal mode coupling and the triggering of NTM by sawteeth. 
In section 2 the equations utilized for our numerical modelling are described. The numerical results for the coupling between the 2/2 and 3/2 modes, obtained from both the single fluid and four-field equations, are presented in Section 3. The results on the triggering of the $3 / 2$ NTMs by sawtooth crashes are presented in Section 4. Finally, the discussions and summary are given in the last section.

\section{Theoretical model}

A circular cross section of the equilibrium magnetic surfaces is assumed. The magnetic field is defined as [26]

$$
B=R_{0}\left[\left(B_{0 t}+b_{t}\right) \nabla \zeta+\nabla \psi \times \nabla \zeta\right]
$$

where $B_{0 t}$ is the vacuum toroidal field at the magnetic axis, $b_{t}$ is due to the diamagnetic correction, $R_{0}$ the major radius of the magnetic axis, $\zeta$ the toroidal angle, and $\psi$ the flux function. The four-field equations, the electron continuity equation, generalized Ohm's law and the equation of motion in the perpendicular (after taking $\nabla \zeta \cdot \nabla x$ ) and the parallel direction, are utilized [26]. Normalizing the length to the plasma minor radius $a$, the time $t$ to the resistive time $\tau_{R}=a^{2} \mu_{0} / \eta$ ( $\eta$ is the plasma resistivity), the magnetic field to $B_{0 t}$, the ion velocity $\boldsymbol{v}$ to $a / \tau_{R}$, and the electron density $n_{e}$ to its value at the magnetic axis, these equations become [26,56,57]

$$
\begin{aligned}
& \frac{d n_{e}}{d t}=d_{1}\left(\nabla_{\|} j-\varepsilon \beta_{e} \frac{\partial\left(n_{e}+\phi / \Omega\right)}{\partial \mathrm{z}}\right)-\nabla_{\|}\left(n_{e} v_{\|}\right)+\nabla \cdot\left(D_{\perp} \nabla n_{e}\right)+S_{n}, \\
& \frac{d \psi}{d t}=E_{0}-\eta\left(j-j_{b}\right)-\frac{\eta}{v_{e i}} \frac{d j}{d t}+\eta \frac{\mu_{e}}{v_{e i}} \nabla_{\perp}^{2} j+\frac{T_{e}}{n_{e}} \Omega \nabla_{\|} n_{e}, \\
& \frac{d U}{d t}=S^{2}\left(\nabla_{\|} j-\varepsilon \beta_{e} \frac{\partial n_{e}}{\partial z}\right)+\mu \nabla_{\perp}^{2} U+\mathrm{S}_{\mathrm{m}}, \\
& \frac{d v_{\|}}{d t}=-C_{s}^{2} \nabla_{\|} p / n_{e}+\mu \nabla_{\perp}^{2} v_{\|} .
\end{aligned}
$$


Differing from Ref. [26], the cold ion assumption is made in Eqs. (2)-(5), while the bootstrap current density $j_{b}$, electron inertia and viscosity (the $3^{\text {rd }}-5^{\text {th }}$ terms in Eq. (3)) are taken into account in Ohm's law. The ion velocity $v=\boldsymbol{v}_{\|}+\boldsymbol{v}_{\perp}$, the subscripts $\|$ and $\perp$ denote the parallel and perpendicular components, $d / d t=\partial / \partial+v_{\perp} \cdot \nabla, j$ is the toroidal plasma current density, $j_{b}=-c_{b} \sqrt{ } \varepsilon\left(\partial p_{e} / \partial r\right) / B_{p}$ the bootstrap current density, $c_{b}$ a constant of order of unity, $\varepsilon=r / R_{0}$ the inverse aspect ratio, $p=p_{e}=n_{e} T_{e}, T_{e}$ the electron temperature, $B_{p}$ the poloidal magnetic field. $U=-\left(R / R_{0}\right)^{2} \nabla_{\perp}^{2} \phi$ is the plasma vorticity, $\phi$ the stream function, $R$ the major radius, $\mathrm{z}$ the coordinate along the vertical direction, $\mu\left(\mu_{e}\right)$ the ion (electron) viscosity, and $D$ the particle diffusivity. $S_{n}$ and $S_{m}$ are the particle and momentum sources, which are choses to maintain specified equilibrium profiles for density and rotation against particle diffusion and viscous drag. $E_{0}$ is the equilibrium electric field, $S=\tau_{R} / \tau_{A}, d_{1}=\omega_{c e} / v_{e i}, \quad \Omega=0.5 \beta_{e} d_{1}$, $C_{s}=\left[T_{e} / m_{i}\right]^{1 / 2} /\left(a / \tau_{R}\right), \quad \beta=\beta_{e}=8 \pi n_{e} T_{e} / B_{0 t}{ }^{2}, \omega_{c e}$ the electron cyclotron frequency, $v_{e i}$ the electron-ion collisional frequency, $\tau_{A}=a / V_{A}$, and the Alfven velocity $V_{A}$ is defined using the toroidal field.

Straight field line coordinates $(r, \theta, \zeta)$ are used with the Jacobian $\sqrt{ } g=r R^{2} / R_{0}$, where $r$ is the minor radius of the equilibrium magnetic surface, and $\theta$ is the "poloidal-like" angle [31]. In this coordinate system the operator $\boldsymbol{B} \cdot \nabla$ takes a simple form. The numerical code TM1 has been upgraded to include the toroidal coupling among modes with the same $n$ but different $m$ numbers, in addition to nonlinear two-fluid effects. The benchmark between TM1 and CASTOR3D [32] indicates that the results from these two codes agree for $n>1$ modes if the $\beta$ value is not too high.

The following equilibrium plasma parameters are used for the reference case: $B_{0 t}=2 T, a=0.5 \mathrm{~m}$, $a / R_{0}=0.2, T_{e}=2 \mathrm{keV}$, and $n_{e}=3 \times 10^{19} \mathrm{~m}^{-3}$, leading to $S=2.6 \times 10^{8}, d_{1}=3.1 \times 10^{7}, C_{s}=2.0 \times 10^{7} \mathrm{a} / \tau_{R}, \tau_{R}=23 \mathrm{~s}$, and $v_{e i}=2.2 \times 10^{4} / \mathrm{s}$. Furthermore, $\mu_{e} / v_{e i}=10^{-4} a^{2}, \mu=0.2 \mathrm{~m}^{2} / \mathrm{s}=19 a^{2} / \tau_{R}, D_{\perp}=\mu / 50$, and a monotonic profile for the safety factor $q$ are assumed with $q_{0}=0.91$ and $r_{q=1}=0.3 a$, where $q_{0}$ is the original equilibrium safety factor at $r=0$, and $r_{q=1}$ the minor radius at the $q=1$ surface. The $q=3 / 2$ surface is at $r_{3 / 2}=0.68 a$. The equilibrium electron density has a radial profile of the form $n_{e}=\left\{0.8\left[1-(r / a)^{2}\right]^{2}+0.2\right\}\left(3 \times 10^{19} m^{-3}\right)$. 
The electron temperature is assumed to be a constant in space and time. These input parameters are used for our numerical calculations if not mentioned elsewhere. Without toroidal mode coupling, the $m / n=2 / 2$ mode is unstable in the linear phase, while the $3 / 2$ mode is stable.

In tokamak experiments the plasma rotation is essentially toroidal, while in Eqs. (2)-(5) due to the large aspect ratio approximation only the poloidal rotation is included, so that an enhanced plasma

viscosity for the $m / n=0 / 0$ component, $\mu_{0 / 0}=19 \times 10^{2}\left(a^{2} / \tau_{R}\right)$, is used in calculations. This value guarantees a reasonable balance between the electromagnetic and viscous force, based on the following considerations [17]: (a) The electromagnetic force in the toroidal direction is smaller by a factor $(n / m)\left(r_{s} / R\right)$ than that in the poloidal direction, where $r_{s}$ is the minor radius of the resonant surface. (b) To have the same mode frequency due to the plasma rotation, the toroidal rotation velocity should be $(\mathrm{m} / \mathrm{n})\left(R / r_{\mathrm{s}}\right)$ times larger than the poloidal one. These two effects lead to a larger viscous force compared to the electromagnetic force for the toroidal rotation case by a factor $\left[(m / n)\left(R / r_{s}\right)\right]^{2}$, which is of the order of $10^{2}$.

\section{Coupling between the $m / n=2 / 2$ and $3 / 2$ modes}

In order to understand the triggering of the $m / n=3 / 2$ mode by a sawtooth crash, it is beneficial to first look into the destabilization of a $3 / 2$ mode by a $2 / 2$ tearing mode without including the $n=1$ perturbations, since the $1 / 1$ mode is an internal kink mode which would grow much more quickly than the $m>1$ tearing modes, and there is no steady $2 / 2$ harmonic during and after sawtooth crashes [47]. In this section the calculations are thus carried out by only including the modes with the helicity $m / n=2 / 2$ and $3 / 2$ as well as the components due to the toroidal mode coupling among $n=2$ components.

\subsection{Calculation results from single fluid equations}


Eqs. (2) - (4) correspond to the single fluid case with $\Omega=d_{1}=C_{s}=\boldsymbol{v}_{\|}=0$ [26-28]. We first study the destabilization of the $3 / 2$ mode by the $2 / 2$ mode in this case. The bootstrap current density is taken to be zero here. The time evolution of the normalized 2/2 and 3/2 island width is shown in Fig. 1 (left) for $\beta_{e}=0, \omega_{2 / 2}=2.02 \times 10^{4} / \tau_{R}, \quad \omega_{3 / 2}=2.37 \times 10^{4} / \tau_{R}, \Delta \omega=\left(\omega_{3 / 2}-\omega_{2 / 2}\right)=3.5 \times 10^{3} / \tau_{R}$, where $\omega_{2 / 2}=2 V_{p} / r$ is the $2 / 2$ mode frequency calculated at $\mathrm{r}_{q=1}, V_{p}$ is an assumed poloidal equilibrium plasma rotation velocity, $\omega_{3 / 2}=3 V_{p} / r$ is the $3 / 2$ mode frequency calculated at $r_{3 / 2}$, and $\Delta \omega$ is the relative frequency between the $3 / 2$ and $2 / 2$ modes, being smaller than the linear $2 / 2$ mode growth rate $\left(\sim 10^{4} / \tau_{R}\right)$. The "island width" $W_{m / n}=4\left[\psi_{m / n} /\left(B_{p} q^{\prime} / q\right)\right]^{1 / 2}$ is calculated at $r_{m / n}$, where $r_{m / n}$ and $\psi_{m / n}$ are the minor radius of the resonant surface and the perturbed flux of the $m / n$ component. It is seen that the $2 / 2$ mode grows first, and the $3 / 2$ mode grows later driven by its coupling to the $2 / 2$ mode. It should be noted that $W_{m / n}$ represents the island width when the magnetic shear is not too small and the "constant $\psi_{m / n}$ " approximation is valid. Otherwise it only gives the change of the value of $\left(\psi_{\mathrm{m} / \mathrm{n}} / q^{\prime}\right)^{1 / 2}$ at $r_{\mathrm{m} / \mathrm{n}}$.
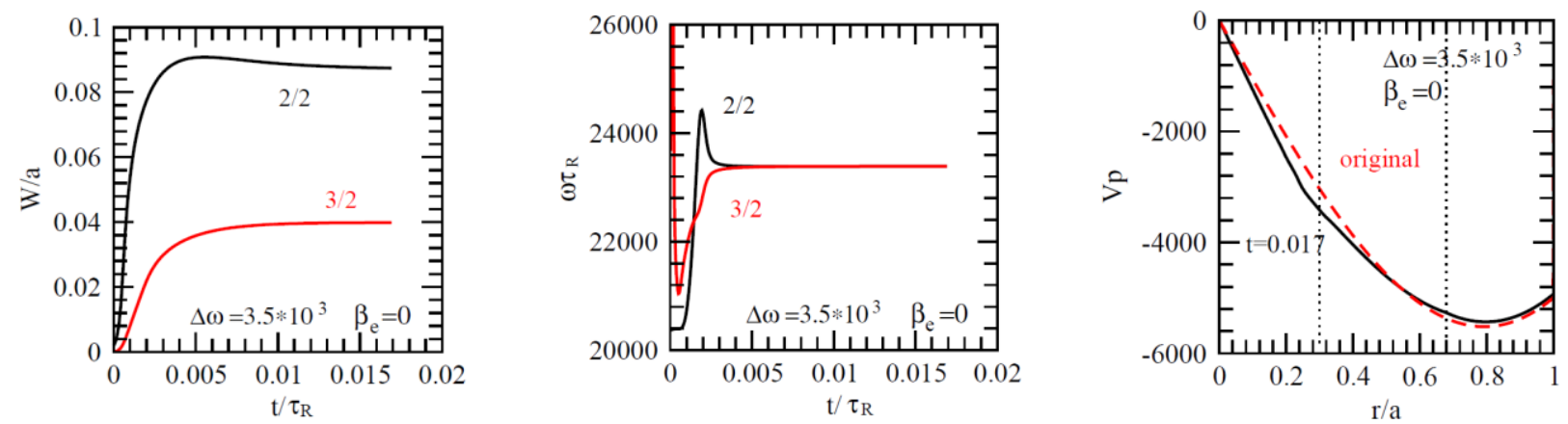

Figure $1 \quad$ Time evolution of $W_{2 / 2} / a$ and $W_{3 / 2} / a$ (left), mode frequencies (middle) and radial profiles of the poloidal plasma rotation velocity at $t=0$ and in steady state (right) for $\beta_{e}=0$ and $\Delta \omega=\left(\omega_{3 / 2}-\omega_{2 / 2}\right)=3.5 \times 10^{3} a / \tau_{R}$. The 3/2 mode grows driven by its coupling to the 2/2 mode. The frequencies of these two modes approach the same value when the island widths are sufficiently large.

The corresponding time evolution of the normalized 2/2 and 3/2 mode frequencies, calculated from the imaginary part of $\left(\partial \psi_{m / n} / \partial\right) / \psi_{m}$, are shown in Fig. 1 (middle). The frequencies of these two modes approach the same value when the island widths are sufficiently large, indicating that the two modes 
are locked together. The corresponding radial profile of the normalized poloidal plasma rotation velocity in steady state is shown in figure 1 (right) by the solid curve. The poloidal rotation speed is increased at $r_{q=1}$ but decreased at $\mathrm{r}_{3 / 2}$ compared to the original equilibrium velocity shown by the dashed curve, such that these two modes can have the same frequencies. The island rotates together with the local plasma once its width is sufficiently large.

Fig. 2 (left) shows the time evolution of the corresponding island widths for a factor of ten increased relative equilibrium rotation frequency $\left(\Delta \omega=3.5 \times 10^{4} / \tau_{R}\right)$ between the $\mathrm{m} / \mathrm{n}=3 / 2$ and the $2 / 2$ modes. This has been achieved by using a stronger momentum source in equation (4), while keeping the other input parameters unchanged. It is seen that again the $2 / 2$ mode grows first, and the $3 / 2$ mode growth is delayed compared to the case of a smaller relative mode frequency shown in figure 1(left). There are small oscillations in the mode amplitude in the nonlinear saturation phase.
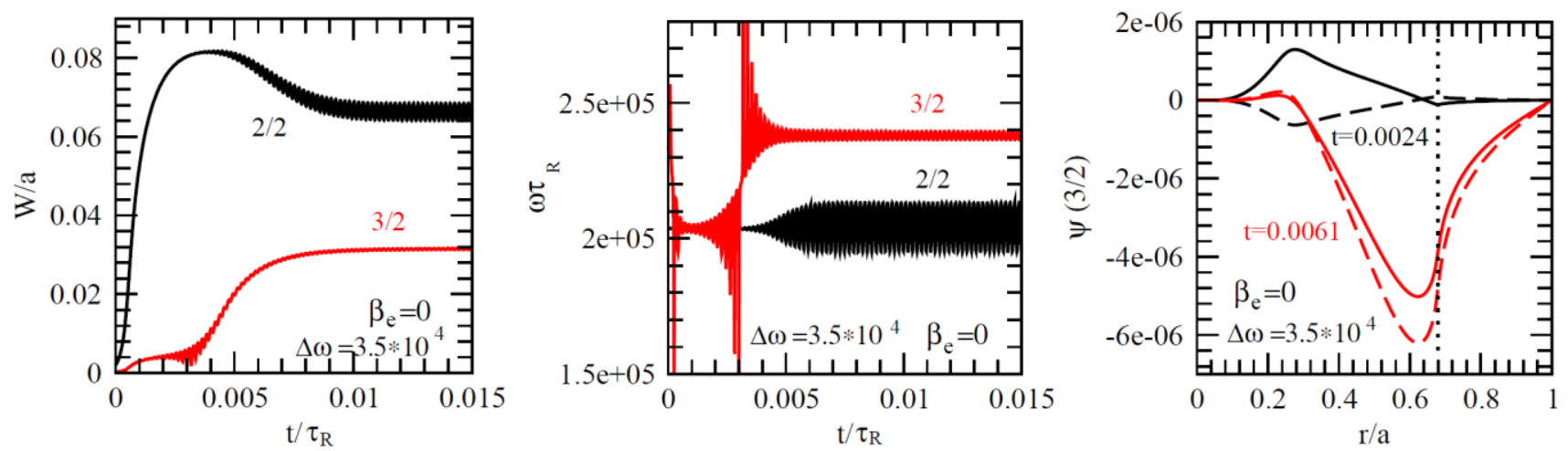

Figure 2 Time evolution of $W_{2 / 2} / a$ and $W_{3 / 2} / a$ (left), mode frequencies (middle) and radial profiles of the normalized flux functions $\psi_{3 / 2}$ (right) at $t=0.0024 \tau_{R}$ (black) and $0.0061 \tau_{R}$ (red) for $\beta_{e}=0$ and $\Delta \omega=3.5 \times 10^{4} / \tau_{R}$. The solid (dashed) curves are for the real (imaginary) parts. The frequencies of these two modes are different once the island widths are sufficiently large. The oscillations in the mode amplitude and frequencies correlate with the change of the relative phase between these two modes. The $\psi_{3 / 2}$ profiles first have the feature of an ideal mode across the $q=3 / 2$ surface marked by the vertical dotted line and then the feature of a tearing mode at later time. 
The corresponding mode frequencies are shown in figure 2 (middle). The two modes have the same frequencies around $t=0.001 \tau_{R}$ when the $3 / 2$ island width is still small. During this phase the $3 / 2$ mode frequency follows that of the $2 / 2$ mode, since the latter is driving the $3 / 2$ mode, and a plasma can slip through a sufficiently small island. Once the $3 / 2$ island is sufficiently large, its frequency is determined by the local plasma rotation frequency at $r_{3 / 2}$, since in this case the island rotates together with the local plasma. The radial profile of the plasma rotation velocity is essentially not changed due to the stronger momentum source. Thus, the $3 / 2$ mode frequency differs from that of the $2 / 2$ mode. The relative frequency between these two modes is not too large, being comparable to the linear $2 / 2$ mode growth rate, so that the $3 / 2$ mode is still driven to grow by its coupling to the $2 / 2$ mode. The oscillations in the mode amplitude and frequencies correlate with the change of the relative phase between these two modes.

Corresponding radial profiles of the $\mathrm{m} / \mathrm{n}=3 / 2$ component of the normalized (to $a B_{0 t}$ ) flux functions, $\psi_{3 / 2}$, are shown in figure 2 (right) at two different times, $t=0.0024 \tau_{R}$ (black) and $0.0061 \tau_{R}$ (red), where the solid (dashed) curves represent the real (imaginary) parts. The $\psi_{3 / 2}$ profiles first have the feature of an ideal mode $t=0.0024 \tau_{R}$, since $\psi_{3 / 2}$ is about zero at $r_{3 / 2}$ (marked by the vertical dotted line), and the 3/2 perturbations outside the $q=3 / 2$ surface are shielded. This effect is similar to the shielding observed in RMP (resonant magnetic perturbation) penetration calculations when the plasma rotation frequency is sufficiently large [56]. Later in time the $\psi_{3 / 2}$ profiles have the feature of a tearing mode when the $3 / 2$ island has grown. 


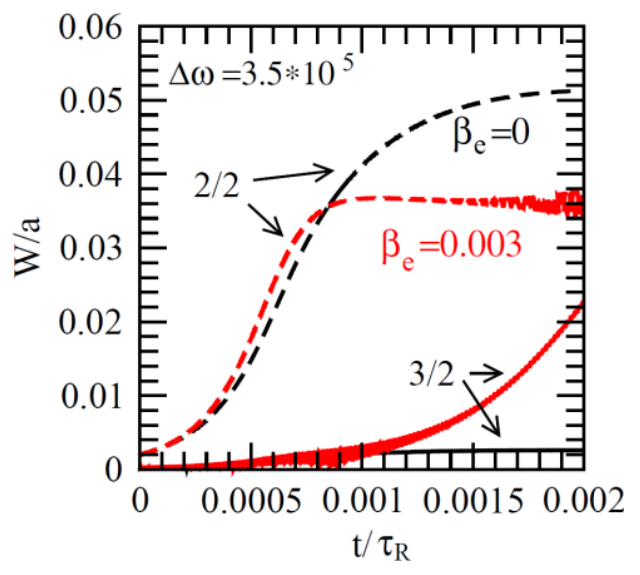

Figure 3 Time evolution of $W_{2 / 2} / a$ (dashed curves) and $W_{3 / 2} /$ a (solid) with $\Delta \omega=3.5 \times 10^{5} / \tau_{R}$ for $\beta_{e}=0$ (black curves) and $\beta_{e}=0.003$ (red). The 3/2 mode is driven to grow by its coupling to the $2 / 2$ mode for a finite $\beta_{e}$.

Further increasing the relative equilibrium mode rotation frequency by ten times to $\Delta \omega=3.5 \times 10^{5} / \tau_{R}$, the time evolution of $W_{2 / 2} / a$ (dashed curves) and $W_{3 / 2} / a$ (solid) is shown in Fig. 3 . It is seen that for $\beta_{e}=0$ (black curves), the $3 / 2$ island width is close to zero, indicating the shielding of mode coupling by a large differential rotation. The $2 / 2$ mode amplitude is also smaller compared to those shown in figures 1 and 2. With $\beta_{e}=0.003$ (red curves), the $3 / 2$ island width is driven to grow by its coupling to the $2 / 2$ mode, indicating that a finite $\beta_{e}$ enhances mode coupling, as seen from Eq. (4). The oscillation in the 2/2 island width (red curve) is again caused by the change in the relative phase between the two modes, which is larger when the 3/2 island becomes larger, since the interaction between the two modes is stronger for larger mode amplitude. The radial profile of the plasma rotation velocity is again not changed. The time evolution of the mode frequencies is similar to that shown in figure 2 . When the $3 / 2$ island is small, the $3 / 2$ mode frequency follows that of the $2 / 2$ mode. Once the $3 / 2$ island is sufficiently large, its frequency is determined by the local plasma rotation frequency at $r_{3 / 2}$, being different from the 2/2 mode frequency.

The above results demonstrate the expected results for a single fluid model: the toroidal mode coupling, leading to the destabilization of the $3 / 2$ mode by the $2 / 2$ mode, is weakened by a larger relative rotation frequency between these two modes but is enhanced by a finite value of $\beta_{e}$. Similar 
results have already been obtained in full toroidal geometry, but without including plasma rotation $[50,51]$.

To conclude, the results of this subsection are summarized in figure 4 , showing the ratio of $W_{3 / 2} / W_{2 / 2}$ in steady state as a function of the frequency difference between the $2 / 2$ and $3 / 2$ mode, $\Delta \omega$, for $\beta_{e}=0$ (black circles). In this case the destabilization of the $3 / 2$ mode by the $2 / 2$ mode is effective only for a sufficiently low value of $\Delta \omega$. The result for $\beta_{e}=0003$ at $t=0.002 \tau_{R}$ is also added in the figure, indicating the destabilizing effect of finite $\beta_{e}$.

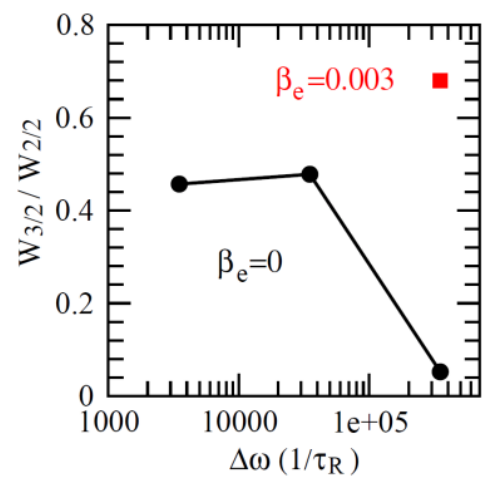

Figure $4 \quad W_{3 / 2} / W_{2 / 2}$ in steady versus the frequency difference between the $2 / 2$ and $3 / 2$ mode, $\Delta \omega$, for $\beta_{e}=0$ (black circles). The result for $\beta_{e}=0003$ at $t=0.002 \tau_{R}$ is shown by the red square.

\subsection{Calculation results from four-field equations}

In the following, the full four-field equations, Equations (2)-(5), are utilized. If not stated otherwise, the bootstrap current density fractions $f_{b}=0.066$ at $r_{q=1}$ and $f_{b}=0.2$ at $r_{3 / 2}$ are taken. First, results on the destabilization of the 3/2 mode with a sufficiently high $\beta_{e}$ value will be presented in Section 3.2.1, followed by the results for a low and moderate $\beta_{e}$ values in Section 3.2.2. The effect of plasma rotation will be shown in Section 3.2.3. The effect of perpendicular particle diffusivity is given in Section 3.2.4, followed by the effect of the electron pressure profile in Section 3.2.5.

\subsubsection{Destabilization of the $3 / 2$ mode}


The electron diamagnetic drift frequency is proportional to the input parameter $\Omega$ in equation (3) for a given electron density profile. The time evolution of $W_{2 / 2} / a$ (dashed curves) and $W_{3 / 2} / a$ (solid) is shown in Fig. 5 (left) for $\Omega=10^{4}$ (black curves), corresponding to $\omega *_{e, 2 / 2}=0.68 \times 10^{5} / \tau_{R}\left(\mathrm{f}_{* e, 2 / 2}=0.47 \mathrm{kHz}\right)$, $\omega_{*_{e}, 3 / 2}=1.2 \times 10^{5} / \tau_{R}\left(f_{*_{e}, 3 / 2}=0.83 \mathrm{kHz}\right)$ and $\Delta \omega_{*_{e}}=\left(\omega_{*_{e, 3 / 2}}-\omega_{*_{e}, 2 / 2}\right)=5.2 \times 10^{4} / \tau_{R}$, where $\omega_{*_{e}, 2 / 2}=2 V_{*_{e}} / r$ is calculated at $r_{q=1}, V_{*_{e}}$ is the equilibrium electron diamagnetic drift velocity, $\omega_{* e, 3 / 2}=3 V_{*_{e}} / r$ is calculated at $r_{3 / 2}$, and $\Delta \omega_{*_{e}}$ is the relative frequency between the $3 / 2$ and $2 / 2$ modes due to the electron diamagnetic drift. It is seen that the $3 / 2$ mode is driven to grow by its coupling to the $2 / 2$ mode already for a quite low $\beta_{e}$ value, $\beta_{e}=5 \times 10^{-4}$, after the $2 / 2$ mode has grown. The $3 / 2$ mode frequency approaches that of the $2 / 2$ mode during mode growth. Increasing the value of $\Omega$ by two times to $\Omega=2 \times 10^{4}$ and for $\beta_{e}=0.001$ (red curves), the time evolution of $W_{2 / 2} / a$ and $W_{3 / 2} / a$ is similar. The $2 / 2$ mode grows first, and the $3 / 2$ mode grows later. However, a larger value of $\Omega$ (diamagnetic drift frequency) results in a slower mode growth, even if the $\beta_{e}$ value is two times larger. Corresponding to the black curves in the left figure, radial profiles of the $m / n=0 / 0$ component electron density at $t=0$ and $0.005 \tau_{R}$ are shown in Fig. 5 (right). The electron density flattens around the $q=1$ and 1.5 surfaces at $t=0.005 \tau_{R}$ due to sufficiently large magnetic islands formed there.
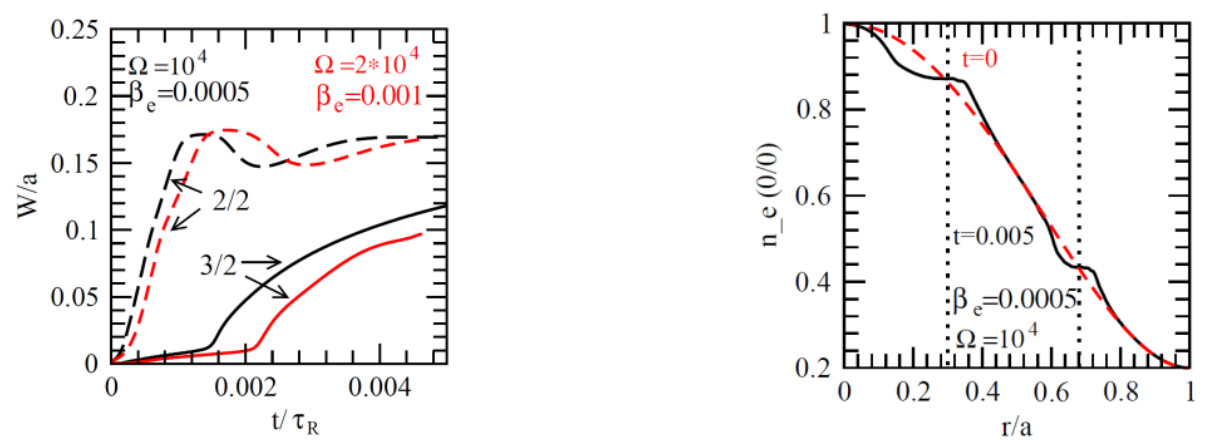

Figure 5 (left) Time evolution of $W_{2 / 2} / a$ (dashed curves) and $W_{3 / 2} / a$ (solid) for $\beta_{e}=0.0005 / \Omega=10^{4}$ (black) and $\beta_{e}=0.001 / \Omega=2 \times 10^{4}$ (red). (right) Radial profiles of the normalized $\mathrm{m} / \mathrm{n}=0 / 0$ component electron density at $t=0$ and 0.005 for $\beta_{e}=0.0005 / \Omega=10^{4}$. The locations of $r_{q=1}$ and $r_{3 / 2}$ are marked by vertical dotted lines. 
For the results shown in figure 5, the “constant $\psi$ ” approximation of the tearing mode is found after the $3 / 2$ mode grows up due to a smaller value of $\Omega$, similar to the red curves shown in figure 2 (right). While before the 3/2 mode grows up, the $\psi_{3 / 2}$ profiles are similar to the black curves in figure 2 (right), and the $3 / 2$ perturbations outside the $\mathrm{q}=3 / 2$ surface are shielded.

Further increasing the diamagnetic drift frequency ( $\Omega$ value), the mode evolution becomes significantly different. An example of the time evolution of $W_{2 / 2} /$ a (dotted curves) and $W_{3 / 2} / a$ (solid) is shown in Fig. 6 (left) for $\Omega=4 \times 10^{4}$ and $\beta_{e}=0.007$. The bootstrap current density fractions are $f_{b}=0.099$ at $r_{q=1}$ and $f_{b}=0.3$ at $r_{3 / 2}$. In this case the $3 / 2$ mode grows first, and its amplitude oscillates in time around $t=3 \times 10^{-4} \tau_{R}$. The $2 / 2$ mode growth is suppressed at the beginning but grows more quickly later. The oscillation in $W_{3 / 2}$ is usually observed in calculations for sufficiently large values of $\Omega$ and $\beta_{e}$. It will be termed as the oscillation regime in the following, while the NTM regime will only refer to the case in which the island grows up to a sufficiently large width.
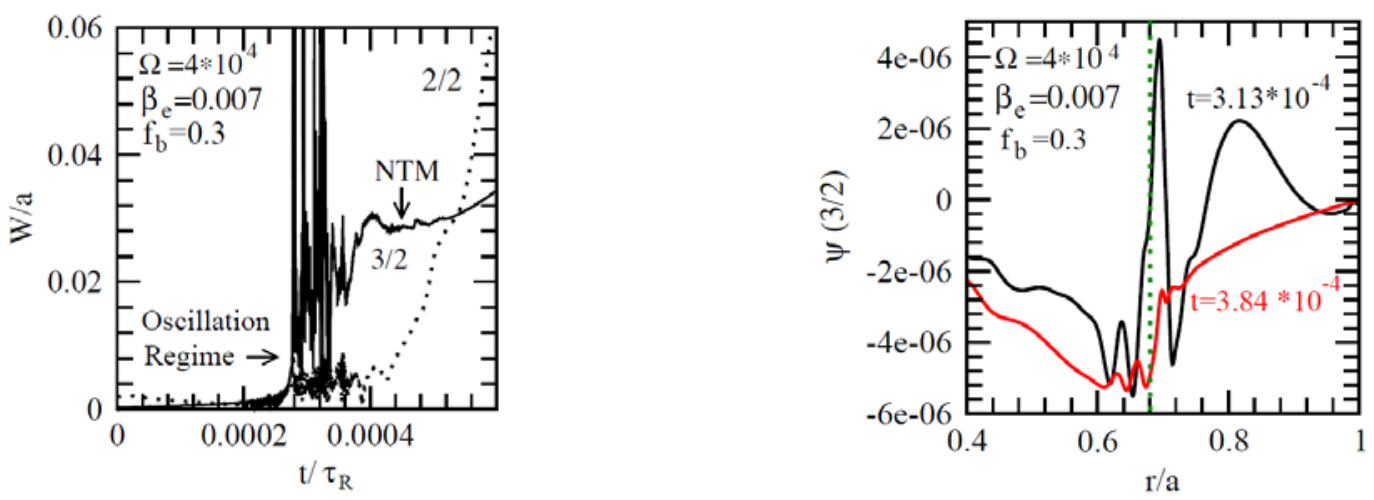

Figure 6 (left) Time evolution of $W_{2 / 2} / a$ (dotted curves) and $W_{3 / 2} / a$ (solid) for $\beta_{e}=0.007 / \Omega=4 \times 10^{4}$. (right) Corresponding radial profiles of the normalized real part of $\psi_{3 / 2}$ at $t=3.13 \times 10^{-4} \tau_{R}$ (black) and $3.84 \times 10^{-4} \tau_{R}$ (red). The $\psi_{3 / 2}$ profiles first have the feature of an ideal mode, since it is close to zero at $r_{3 / 2}$ marked by the vertical dotted line. In a later time the $\psi_{3 / 2}$ profiles have the feature of a tearing mode when the 3/2 island grows up.

Corresponding to figure 6 (left), the radial profiles of the normalized real part of flux functions $\psi_{3 / 2}$ are shown in figure 6 (right) at two different times, $t=3.13 \times 10^{-4} \tau_{R}$ (black curve) and $3.84 \times 10^{-4} \tau_{R}$ (red). 
The profiles of imaginary part are similar to that of the real part. The $\psi_{3 / 2}$ profiles first have the feature of an ideal mode, since the value of $\psi_{3 / 2}$ is small at $r_{3 / 2}$ (marked by the vertical dotted line). However, the $n=2$ perturbations are not shielded by the $q=3 / 2$ surface. In a later time the $\psi_{3 / 2}$ profiles take the feature of a tearing mode, although the $\psi_{3 / 2}$ profile is not smooth across $r_{3 / 2}$. The amplitudes of $\psi_{3 / 2}$ outside the $q=3 / 2$ surface are of the same order of magnitude at the two different times but they significantly differ at $r_{3 / 2}$. Such a feature was observed in experiments [10].

When the 3/2 mode grows up into the NTM regime, the electron density flattens around the corresponding resonant surface as e.g. shown in figure 5 (right). When the $3 / 2$ mode is in the oscillation regime, however, the radial profiles of the plasma current density and electron density are significantly different. Corresponding to figure 6 (left), figure 7 shows the radial profiles of the $m / n=0 / 0$ component plasma current density (left), the safety factor (middle), and the $0 / 0$ component electron density (right) at $t=0$ and $3.13 \times 10^{-4} \tau_{R}$. There is a strong perturbation in the local plasma current density around the $q=3 / 2$ surface due to mode coupling and low plasma resistivity, similar to that observed in the calculations of plasma response to externally applied RMPs [57]. The safety factor is calculated from $q=r B_{t} /\left(R B_{p, 0 / 0}\right)$, where $B_{p, 0 / 0}$ is the $m / n=0 / 0$ component poloidal field. The flattening of the local q-profile around the $q=3 / 2$ surface is due to the change of the local plasma current density, resulting in the sparks in $W_{3 / 2}$ around $t=3 \times 10^{-4} \tau_{R}$ in figure 6 (left) since $W_{3 / 2} \sim 1 / q^{\prime}$, despite that the amplitude of $\psi_{3 / 2}$ is small at $r_{3 / 2}$ as shown in figure 6 (right). There is a drop in the local electron density at $r_{3 / 2}$ marked by the vertical dotted line, while the electron density increases on its two sides. The perturbations in the local plasma current density and electron density around the $q=3 / 2$ surface are usually found in the oscillation regime. 

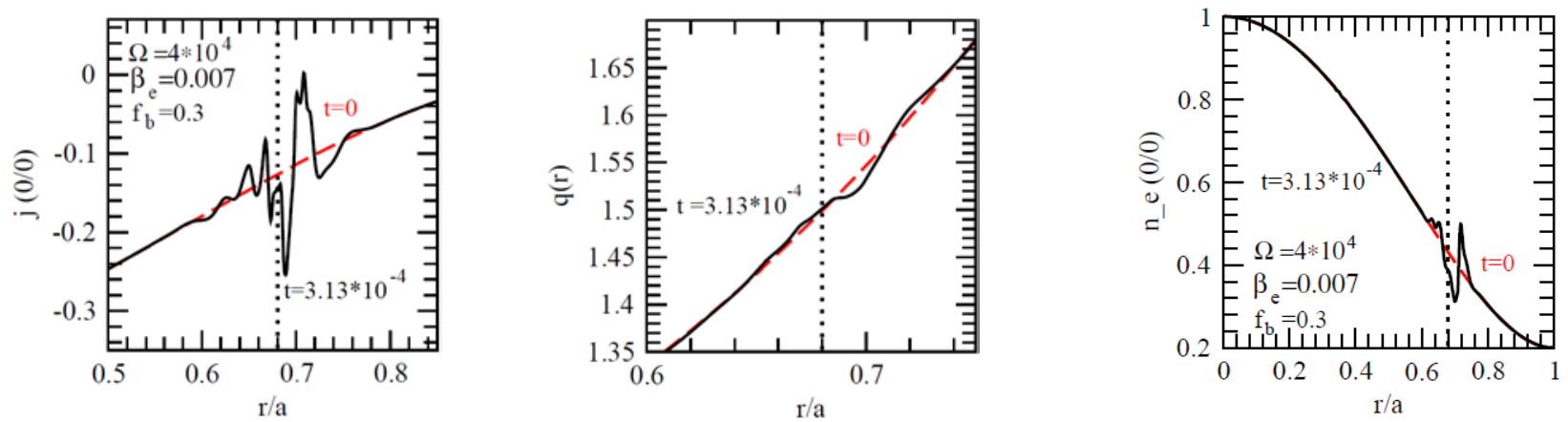

Figure $7 \quad$ Corresponding to figure 6, radial profiles of the $m / n=0 / 0$ component plasma current density (left), the safety factor (middle), and the $\mathrm{m} / \mathrm{n}=0 / 0$ component electron density (right) at $t=0$ and $3.13 \times 10^{-4} \tau_{R}$.

In the oscillation regime a mode with a frequency being much larger than the equilibrium electron diamagnetic drift frequency is generally observed from the spectrogram of magnetic flux perturbation, as shown in figure 8 (left), corresponding to the case of figure 6 . This is caused by the interaction of modes of different frequency as well as the shear Alfven resonance. Corresponding radial profiles of the shear Alfven frequency, $\omega_{S A}=k_{\|} V_{A}$, and the diamagnetic drift frequency for $m=3$ mode, $\omega_{*} e, 3=$ $3 V_{*_{e}} / r$, at $t=3.1 \times 10^{-4} \tau_{R}$ are shown in figure 8 (right), where $k_{\|}=(\mathrm{m} / \mathrm{r})\left(1-q / q_{s}\right) B_{p, 0 / 0} / B_{0 t}$ is the parallel wave vector of the $3 / 2$ mode and calculated from the $m / n=0 / 0$ component magnetic field, $q_{s}$ is the $q$ value at the $q=3 / 2$ surface, and $V_{*_{e}}$ is the electron diamagnetic drift velocity calculated using the $0 / 0$ component electron density. It is seen that these two frequencies are close around $\left|r-r_{3 / 2}\right| \sim 0.03 a$. This feature is commonly observed in the oscillation regime. A local change in the electron density gradient, such as that shown in figure 7 , results in a larger diamagnetic drift frequency such that it can reach $\omega_{S A}$ outside the linear tearing layer. The linear tearing layer width is smaller than 0.01a. It is known from the single fluid theory that the shear Alfven resonance can cause significant perturbations in plasma current density and vorticity if the condition $\omega_{S A}=\omega_{p}$ is satisfied outside the tearing layer [58-61], where $\omega_{p}$ is the plasma rotation frequency for a mode. When the local magnetic shear is significantly decreased in the oscillation regime, the shear Alfven resonance is more easily satisfied, since $k_{\|} \sim$ $\left(q^{\prime} / q_{s}\right)$ near the resonant surface. 

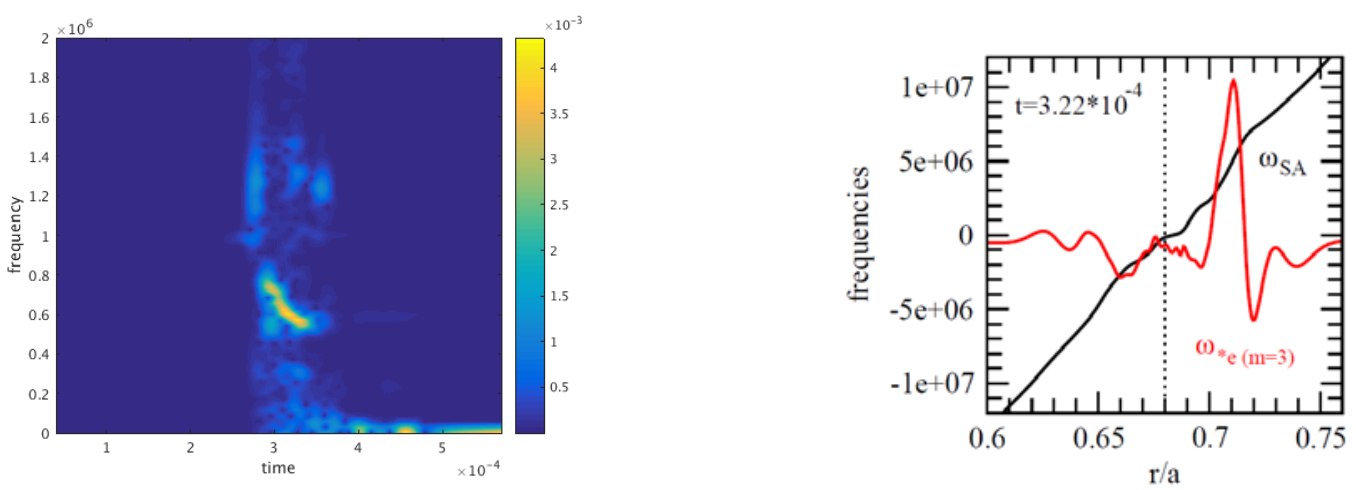

Figure 8 Corresponding to figure 6 for $\beta_{e}=0.007 / \Omega=4 \times 10^{4}$, (left) the spectrogram of the magnetic flux perturbation at $r=0.917$ a. There is a mode fluctuating around the frequency $f \sim 10^{6} / \mathrm{t}_{R} \sim 43 \mathrm{kHz}$ in the oscillation regime. The mode frequency approaches zero after entering the NTM regime. (right) radial profiles of the shear Alfven frequency and the $m=3$ diamagnetic drift frequency in oscillation regime at $3.22 \times 10^{-4} t_{R}$.

Calculations have also been carried out by using a sufficiently large (small) value of $V_{A}(\Omega)$ while keeping other input parameters unchanged. In these cases no high frequency mode is observed, and $\omega_{S A}$ $=\omega *_{e, 3}$ only at $\left|r-r_{3 / 2}\right| \sim 0$, indicating that the high frequency mode is caused by the shear Alfven resonance.

With an even larger value of $\Omega$, the results are similar to those shown in figures 6-8 for a sufficiently large value of $\beta_{e}$. If the bootstrap current density is sufficiently low, the $3 / 2$ mode saturates in the oscillation regime. Taking $C s=0$, the results are about the same. Increasing the value of $d_{1}$ in equation (2), the $3 / 2$ mode grows into the oscillation regime already at a lower $\beta_{e}$ value.

The results shown above indicate that in the framework of the four-field equations, the destabilization of the $3 / 2$ mode by its coupling to the $2 / 2$ mode is significantly affected by the value of $\Omega$ (electron diamagnetic drift frequency). For a lower value of $\Omega$, the $3 / 2$ mode grows into the $N T M$ regime even for a quite low $\beta_{e}$. Increasing the value of $\Omega$, the $3 / 2$ mode is destabilized at a larger $\beta_{e}$ value. In this case the $3 / 2$ mode first grows into the oscillation regime. In this regime the $\psi_{3 / 2}$ profiles 
have the feature of an ideal mode at the $3 / 2$ rational surface, but the $n=2$ perturbations are not shielded by $q=3 / 2$ surface. In a later time the 3/2 mode enters the NTM regime if the bootstrap current density is sufficiently large.

Comparing the results obtained from the single fluid equations shown in figures 3 to those from the four-field equations in figure 6 (left), it is found that the $3 / 2$ mode is more easily destabilized by the $2 / 2$

mode in the framework of the single fluid equations. The value of $\Delta \omega\left(=3.5 \times 10^{5} / \tau_{R}\right)$ in figure 3 is larger than that of $\Delta \omega *_{e}\left(=2.08 \times 10^{5} / \tau_{R}\right)$ for $\Omega=4 \times 10^{4}$ in figure 6 , while the $3 / 2$ mode is destabilized for the single fluid case at a lower $\beta_{\mathrm{e}}$ value. This indicates that the diamagnetic drift and the associated ion polarization current are stabilizing for the island growth, as expected from existing theories [35-41] and seen from the two-fluid calculation results without including mode coupling $[42,43]$.

To conclude this sub-section: With increasing electron diamagnetic drift frequency, a larger $\beta_{e}$ value is required for destabilizing the $3 / 2$ mode by its coupling to the $2 / 2$ mode. For sufficiently large values of $\Omega$ and $\beta_{e}$, the $3 / 2$ mode first grows into the oscillation regime and has the feature of an ideal mode, which enters the NTM regime later if the bootstrap current density is sufficiently large.

\subsubsection{Mode suppression at low $\beta_{e}$}

For a sufficiently low $\beta_{e}$ but a high value of $\Omega$, the growth of the originally unstable $2 / 2$ mode is found to be suppressed by its coupling to the $3 / 2$ mode. With $\Omega=4 \times 10^{4}$, the time evolution of $W_{2 / 2} /$ a (dotted curves) and $W_{3 / 2} / a$ (solid) is shown in Fig. 9 (left) for $\beta_{e}=0.001$ (solid curve). The dashed curve is for the $2 / 2$ mode alone (i.e., the toroidal mode coupling is not included in the calculation). The $2 / 2$ mode grows more slowly than that without the mode coupling, and the 3/2 mode saturates at a small amplitude. 

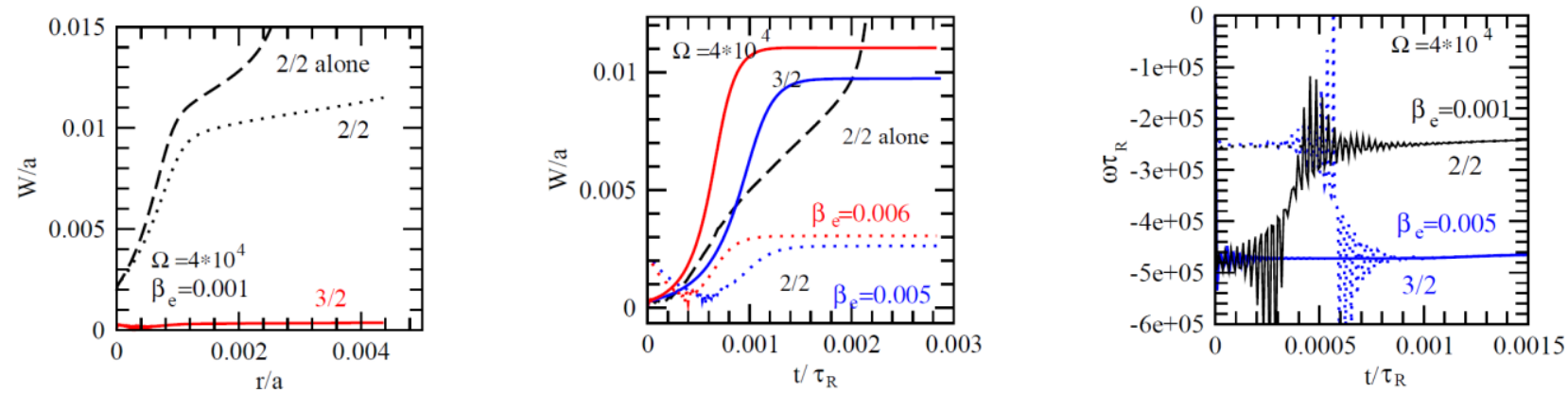

Figure 9 With $\Omega=4 \times 10^{4}$, (left) Time evolution of $W_{2 / 2} / a$ (dotted curves) and $W_{3 / 2} / a$ (solid) for $\beta_{e}=0.001$.

The dashed curve is for the 2/2 mode alone, i.e. without mode coupling. (middle) Time evolution of $W_{2 / 2} /$ a (dotted curves) and $W_{3 / 2} / a$ (solid) for $\beta_{e}=0.005$ (blue) and 0.006 (red). The dashed curve is for the 2/2 mode alone. (right) Time evolution of the normalized 2/2 (dotted curves) and 3/2 (solid) mode frequencies for $\beta_{e}=0.001$ (black) and 0.005 (blue).

In figure 9 (middle) the time evolution of $W_{2 / 2} /$ a (dotted curves) and $W_{3 / 2} / a$ (solid) are shown for higher $\beta_{e}$ values, $\beta_{e}=0.005$ (blue) and 0.006 (red), being slightly lower than that for figure 6 (left). The case for the 2/2 mode alone is also shown (black dashed). The 3/2 island saturates at a small width of about $0.01 a$ but is larger than that of the $2 / 2$ mode. It is interesting to note that the $2 / 2$ mode growth is suppressed by its coupling to the $3 / 2$ mode in this case. In order to understand these results, it is helpful to consider the effect of externally applied RMPs on NTMs [57]. There it was found that if the local electron diamagnetic drift frequency is sufficiently large, the NTM growth can be suppressed by RMPs of moderate amplitude [57]. A similar physics mechanism seems to also exist in the mode coupling process studied here.

In Fig. 9 (right) the time evolution of the normalized 2/2 (dotted curves) and 3/2 (solid) mode frequencies are shown for $\beta_{e}=0.001$ (blue) and 0.005 (red). The $3 / 2$ mode frequency approaches that of the $2 / 2$ mode for $\beta_{e}=0.001$. For the case with $\beta_{e}=0.005$ or 0.006 , however, the frequency of the $2 / 2$ mode approaches that of the $3 / 2$ mode. 


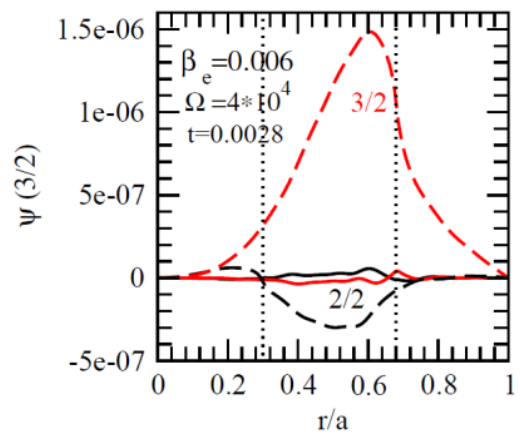

Figure $10 \quad$ Corresponding to figure 9 (middle), radial profiles of the normalized $\psi_{2 / 2}$ (black) and $\psi_{3 / 2}$ (red) for $\Omega=4 \times 10^{4}$ and $\beta_{e}=0.006$ at $t=2.8 \times 10^{-3} \tau_{R}$. The solid (dashed) curves are for the real (imaginary) parts. The locations of $q=1$ and 3/2 surfaces are marked by the vertical dotted lines. The $\psi_{2 / 2}$ profile has the feature of an ideal mode, while the $\psi_{3 / 2}$ profile has the feature of a tearing mode.

With $\Omega=4 \times 10^{4}$ and $\beta_{e}=0.001$, the $\psi_{2 / 2}$ profiles have the feature of a tearing mode, and the $\psi_{3 / 2}$ profiles have the feature of an ideal mode, so that the $3 / 2$ mode follows the 2/2 mode frequency. Corresponding to figure 9 (middle) with $\Omega=4 \times 10^{4}$ and $\beta_{e}=0.006$, radial profiles of the normalized $\psi_{2 / 2}$ (black) and $\psi_{3 / 2}$ (red) at $t=2.8 \times 10^{-3} \tau_{R}$ are shown in figure 10 , where the solid (dashed) curves are for the real (imaginary) parts. In this case the $\psi_{2 / 2}$ profile has the feature of an ideal mode, while the $\psi_{3 / 2}$ profile has the feature of a tearing mode, explaining why the $2 / 2$ mode follows the $3 / 2$ mode frequency.

The coupling between tearing and twisting modes was analyzed before, showing that it can be important in addition to the coupling between tearing modes [18].

To conclude, the results of this sub-section indicate that for a sufficiently large diamagnetic drift frequency and moderate $\beta_{e}$ value, the mode coupling can be stabilizing for an originally unstable tearing mode, and this mode becomes a twisting-like (ideal) mode.

\subsubsection{Effect of plasma rotation}

With $\beta_{e}=0.001$ and $\Omega=2 \times 10^{4}$, the effect of plasma rotation on mode coupling is studied in the following. The time evolution of $W_{2 / 2} / a$ (dashed curves) and $W_{3 / 2} / a$ (solid) is shown in Figure 11 (left) 
for different equilibrium plasma rotation frequencies, corresponding to a $2 / 2$ mode frequency $\omega_{E 0}=0$ (black), $1.6 \times 10^{5} / \tau_{R}$ (green), $3.2 \times 10^{5} / \tau_{R}$ (blue), and $-3.2 \times 10^{5} / \tau_{R}$ (red) due to plasma rotation (electric drift). The equilibrium plasma rotation is driven by the momentum source in equation (4). A positive (negative) value of $\omega_{\mathrm{E}}$ refers to the rotation in the electron (ion) drift direction. The equilibrium plasma rotation frequency at $r_{3 / 2}$ is zero. The $2 / 2$ island growth is similar for all these cases, while the $3 / 2$ mode grows only for a small relative rotation frequency between the two rational surfaces $\left(\omega_{E 0}=0\right.$ and $1.6 \times 10^{5} / \tau_{R}$ ). For the case $\omega_{E 0}=1.6 \times 10^{5} / \tau_{R}$, the $3 / 2$ island grows faster than that for $\omega_{E 0}=0$. The mode frequency results from a combination of the plasma rotation and electron diamagnetic drift. For the green curve the relative frequency due to plasma rotation, $\Delta \omega_{E 0}=\left(\omega_{E 0,3 / 2}-\omega_{E 0,2 / 2}\right)=-1.6 \times 10^{5} / \tau_{R}$, is close to that due to diamagnetic drift, $\Delta \omega_{*}=1.04 \times 10^{5} / \tau_{R}$, but with opposite sign. Thus, the relative electron fluid velocity and the relative mode frequency are the lowest for this case.
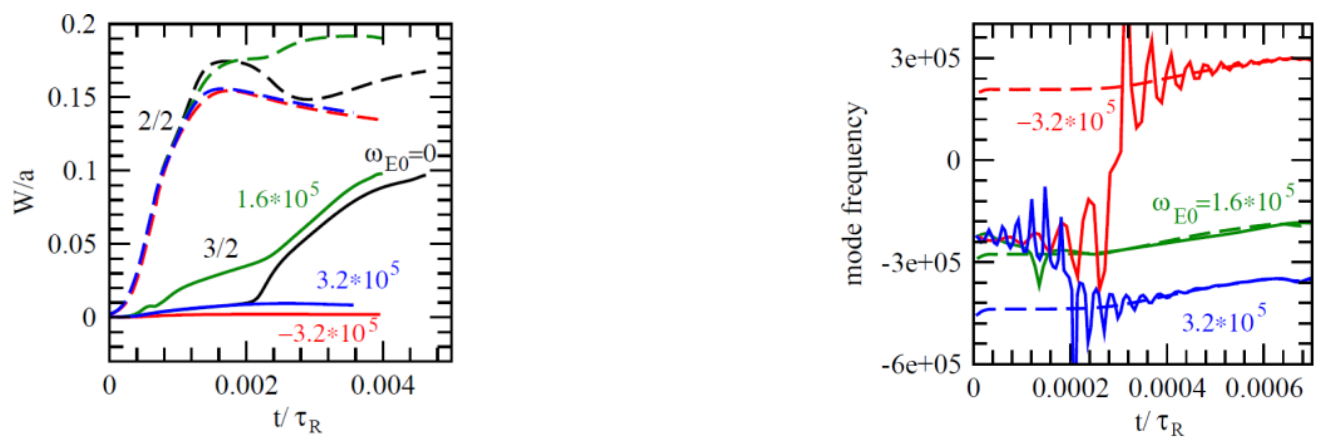

Figure 11 (left) Time evolution of $W_{2 / 2} / a$ (dashed curves) and $W_{3 / 2} / a$ (solid) with $\beta_{e}=0.001 / \Omega=2 \times 10^{4}$ for different ExB rotation frequencies of the 2/2 mode, $\omega_{E 0}=0$ (black), $1.6 \times 10^{5}$ (green), $3.2 \times 10^{5}$ (blue), and $3.2 \times 10^{5} / \tau_{R}$ (red). The positive (negative) value refers to the rotation in the electron (ion) drift direction. (right) corresponding time evolution of the $2 / 2$ (dashed curves) and $3 / 2$ (solid) mode frequencies for $\omega_{\mathrm{E} 0}=1.6 \times 10^{5}$ (green), $3.2 \times 10^{5}$ (blue) and $-3.2 \times 10^{5} / \tau_{R}$ (red). 
The time evolution of mode frequencies are shown in figure 11 (right) for $\omega_{\mathrm{E} 0}=1.6 \times 10^{5} / \tau_{R}$ (green), $3.2 \times 10^{5} / \tau_{R}$ (blue) and $-3.2 \times 10^{5} / \tau_{R}$ (red). In all cases, the $3 / 2$ mode frequency approaches that of the $2 / 2$ mode.

To conclude, the results of this sub-section indicate that the mode coupling is stronger for a lower relative mode frequency determined by the local electron fluid velocity and mode numbers.

\subsubsection{Effect of perpendicular particle diffusivity}

The perpendicular transport is known to affect the NTM growth. Increasing the perpendicular particle diffusivity by 10 times to $D_{\perp}=3.76 a^{2} / \tau_{R}$ and using $\Omega=4 \times 10^{4}, f_{b}=0.099$ at $r_{q=1}$ and $f_{b}=0.3$ at $r_{3 / 2}$, the time evolution of $W_{2 / 2} /$ a (red curve) and $W_{3 / 2} / a$ (black) is shown for $\beta_{e}=0.008$ in Fig. 12 . The 3/2 mode first grows into the oscillation regime and then $N T M$ regime after $\mathrm{t}=0.0005 \tau_{R}$, similar to the results obtained with a smaller value of $D_{\perp}$. Compared to figure 6 it is found that for a larger value of $D_{\perp}$, a higher $\beta_{e}$ value is required for the $3 / 2$ mode to grow up. In the oscillation regime, the local perturbations in the electron density and plasma current density are also observed, similar to that shown in figure 7 with a smaller value of $D_{\perp}$ except for a larger radial width of the perturbation due to a larger value of $D_{\perp}$. Further increasing the perpendicular particle diffusivity by 5 times to $D_{\perp}=18.8 a^{2} / \tau_{R}$ while keeping the other input parameters unchanged, an even higher $\beta_{e}$ is required for the $3 / 2$ mode to grow. This is consistent with the results in [43] that a larger value of $D_{\perp}$ is stabilizing for island growth.

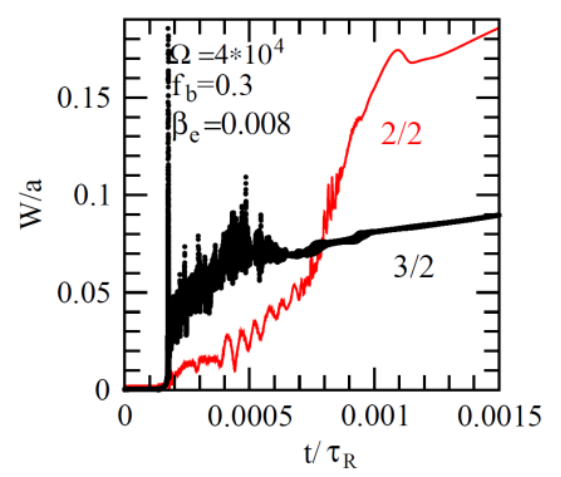


Figure 12 Time evolution of $W_{2 / 2} / a$ (red curve) and $W_{3 / 2} / a$ (black) for $D_{\perp}=3.76 a^{2} / \tau_{R}, \beta_{e}=0.008, \Omega=4 \times 10^{4}$ and $f_{b}=0.3$ at $r_{3 / 2}$.

To conclude, the results in this sub-section indicate that a larger perpendicular particle diffusivity results in a higher $\beta_{e}$ value required for destabilizing the $3 / 2$ mode.

\subsubsection{Effect of electron pressure profile}

In addition to the electron density profile and the value of $\Omega$, it is seen from Ohm's law that the term $\mathrm{T}_{\mathrm{e}} / \mathrm{n}_{\mathrm{e}}$ in front of the parallel electron density gradient affects the local electron diamagnetic frequency. A constant electron temperature in space has been used in all other parts of this paper. In this subsection, however, a radial profile of the equilibrium electron temperature, $T_{e} \sim\left[1-(r / a)^{2}\right]^{2}$, is used, resulting in a smaller value of $\omega_{*}^{*, 3 / 2}$ for same the $\Omega$ value compared to the cases considered before. For $\Omega=9.4 \times 10^{4}$, one finds $\omega_{* e, 2 / 2}=5.3 \times 10^{5} / \tau_{R}, \omega_{* e, 3 / 2}=3.3 \times 10^{5} / \tau_{R}$ and $\Delta \omega_{*}=-2 \times 10^{5} / \tau_{R}$. The value of $\left|\Delta \omega_{*}\right|$ is close to the case $\Omega=4 \times 10^{4}$ in previous sections, but $\omega_{*_{e}, 3 / 2}$ is smaller. With $f_{b}=0.054$ at $r_{q=1}$ and $f_{b}=0.058$ at $r_{3 / 2}$, the time evolution of $W_{2 / 2} / a$ and $W_{3 / 2} / a$ is shown in Fig. 13 for $\beta_{e}=0.005$. It is seen that the growth of the $2 / 2$ and $3 / 2$ mode is similar to that with $\Omega=10^{4}$ and $2 \times 10^{4}$ shown in figure 5, indicating that the $3 / 2$ mode is more easily destabilized by taking into account the radial profile of the electron temperature. The value of $\omega_{*} *_{3 / 2}\left(=3.3 \times 10^{5} / \tau_{R}\right)$ for figure 13 is smaller than that for figure 6 with $\Omega=4 \times 10^{4}\left(\omega_{*, 3 / 2}=4.8 \times 10^{5} / \tau_{R}\right)$. This indicates again that a large local diamagnetic drift frequency at $r_{3 / 2}$ counteracts $3 / 2$ mode destabilization by the $2 / 2$ mode.

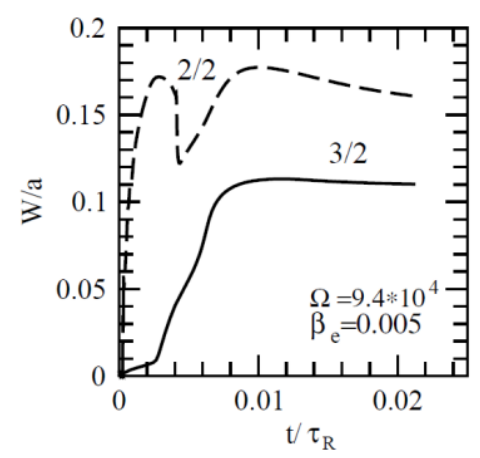


Figure 13 Time evolution of $W_{2 / 2} / a$ (dashed curves) and $W_{3 / 2} / a$ (solid) with $\beta_{e}=0.005 / \Omega=9.4 \times 10^{4} / \tau_{R}$ for $T_{e} \sim\left[1-(r / a)^{2}\right]^{2}$.

To conclude, the results of this sub-section indicate that a large local diamagnetic drift frequency at $r_{3 / 2}$ is more important than the relative mode frequency in preventing the $3 / 2$ mode destabilization by the $2 / 2$ mode.

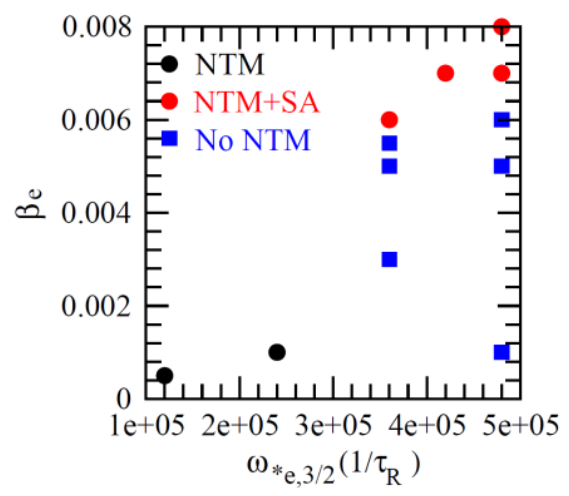

Figure $14 \quad$ Stability diagram in the $\left(\omega_{*, 3 / 2}-\beta_{e}\right)$ plane for the destabilization of the 3/2 mode by the 2/2 mode. The black circles (blue squares) correspond to the cases with (without) 3/2 NTMs. The red circles correspond to cases in which the 3/2 mode is destabilized first into the oscillation regime with shear Alfven resonance and later into the NTM regime. The required $\beta_{e}$ value for destabilizing the 3/2 mode significantly increases for a sufficiently large electron diamagnetic drift frequency at $r_{3 / 2}$.

The results of Section 3.2 are summarized in the $\left(\omega_{* e, 3 / 2}-\beta_{e}\right)$ plane shown in figure 14 , including only the results of Section 3.2.1 and 3.2.2 for simplicity. The black circles (blue squares) correspond to the cases with (without) 3/2 NTMs. The red circles correspond to cases that the $3 / 2$ mode is destabilized first into the oscillation regime with shear Alfven resonance and later into the NTM regime. The required $\beta_{e}$ value for destabilizing the $3 / 2$ mode significantly increases for a sufficiently large electron diamagnetic drift frequency at $r_{3 / 2}$.

\section{Triggering of $m / n=3 / 2$ mode by sawtooth crash}


In this section the $m / n=1 / 1$ mode and its harmonics, $2 / 2,3 / 3, \ldots$, are included in the calculations, in addition to the perturbations with $m / n=3 / 2$ helicity. This will lead to the growth of the internal kink mode and finally to a sawtooth crash $[47,48]$. To isolate effects in our study, toroidal mode coupling is only included among $n=2$ components.

An example of a sawtooth collapse is shown in Fig. 15 for $\Omega=2 \times 10^{4}, \beta_{e}=0.005$, and $f_{b}=0.13$ at $r_{q=1}$ and $f_{b}=0.4$ at $r_{3 / 2}$. The sawtooth happens at $t=2 \times 10^{-5} \tau_{R}$, as seen from the sudden decrease (increase) of the normalized $m / n=0 / 0$ component of the electron density inside (outside) the original $q=1$ surface at $r_{q=1}=0.3 a$ in Fig. 15 (left). The q-profile flattens to about unity in the central region after the collapse as shown in Fig. 15 (middle). There is a large amplitude 2/2 perturbation around the sawtooth crash time at $t=2 \times 10^{-5} \tau_{R}$ (correlating with the $1 / 1$ perturbation amplitude), but it decays shortly afterwards as seen from Fig. 15 (right). Thus, the major difference from the cases discussed in Sec. 3 is in particular that the $2 / 2$ mode responsible for the triggering of the $3 / 2$ mode has a significant large amplitude only for a short time period. Nevertheless, surviving 2/2 postcursor can have a sufficient amplitude to trigger a 3/2 mode.
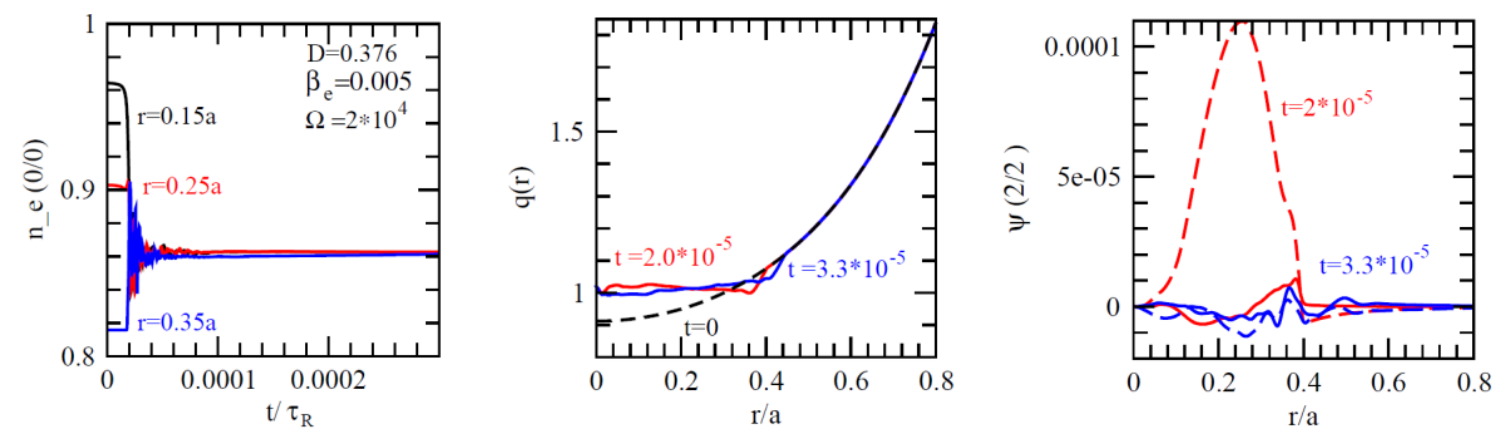

Figure 15 (left) Time evolution of the normalized $m / n=0 / 0$ component electron density at $r=0.15,0.25$ and 0.35a. The sawtooth crash happens at $t=2 \times 10^{-5} \tau_{R}$. (middle) Corresponding q-profiles at $t=0,2.0 \times 10^{-5}$ and $3.3 \times 10^{-5} \tau_{R}$. (right) Corresponding radial profiles of the normalized $\psi_{2 / 2}$ at $t=2.0 \times 10^{-5}$ and $3.3 \times 10^{-5} \tau_{R}$. The solid (dashed) curves are the real (imaginary) parts. 
For the sawtooth crash shown in figure 15 , the time evolution of $W_{3 / 2} / a$ is shown by the black curve in Fig. 16 (left). After sawtooth crash, the 3/2 mode first grows into the oscillation regime around $t=10^{-}$ ${ }^{4} \tau_{R}$ and then into the $N T M$ regime after $2 \times 10^{-4} \tau_{R}$. The red curve is for $\beta_{e}=0.004$, keeping other input parameters the same as those for the black curve. The $3 / 2$ mode remains at a low level for $\beta_{e}=0.004$, showing the destabilizing effect of higher $\beta_{e}$ value similar to what was found in Section 3 without the $1 / 1$ mode.
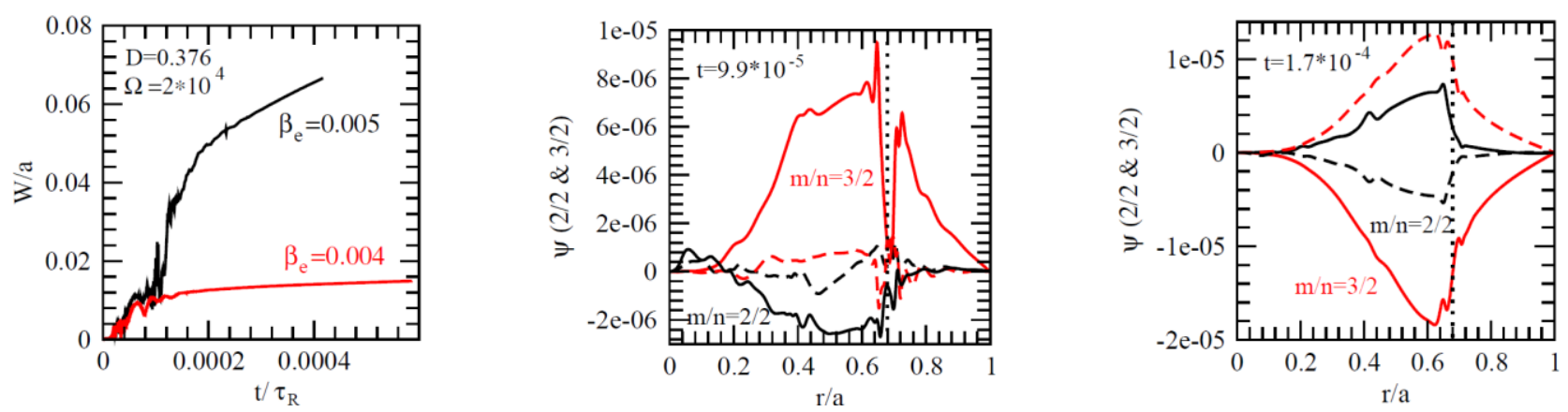

Figure 16 (left) The black curve shows the time evolution of $W_{3 / 2} / a$ corresponding to the parameters of figure 15. The red curve is for $\beta_{e}=0.004$, keeping other input parameters the same as those for the black curve. (middle and right) Corresponding to the black curve in the left figure, radial profiles of the normalized $\psi_{3 / 2}$ (red curves) and $\psi_{2 / 2}$ (black) at $t=9.9 \times 10^{-5} \tau_{R} \sim 2.3 \mathrm{~ms}$ and $1.7 \times 10^{-4} \tau_{R} \sim 3.9 \mathrm{~ms}$. The solid (dashed) curves are the real (imaginary) parts. The vertical dotted line shows the $r_{3 / 2}$ location.

For the parameters of Fig. 15 (corresponding to the black curve in Fig 16 (left)), radial profiles of $\psi_{3 / 2}$ (red curves) and $\psi_{2 / 2}$ (black) are shown at $t=9.9 \times 10^{-5} \tau_{R} \sim 2.3 \mathrm{~ms}$ and $1.7 \times 10^{-4} \tau_{R} \sim 3.9 \mathrm{~ms}$ in figure 16 (middle and right). The solid (dashed) curves are the real (imaginary) parts. The vertical dotted line shows the $r_{3 / 2}$ location. The $\psi_{3 / 2}$ profiles first have the feature of an ideal mode in the oscillation regime, with the $n=2$ perturbations not being shielded by the $q=1.5$ surface. Later in time, when the mode amplitude has become large, the $\psi_{3 / 2}$ profiles show the features of a tearing mode, similar to what was found in Section 3. 
Comparing with the red curves shown in figure 5 (left) in Section 3, which is also obtained for $\Omega=2 \times 10^{4}$ but for a $2 / 2$ island as driver, it is found that a higher $\beta_{e}$ value is required for the $3 / 2$ island to grow up into the NTM regime by a sawtooth crash. The sawtooth crash flattens the central electron density profile in a short time scale ( $\sim 50 \mu$ ), resulting a larger relative frequency between the $2 / 2$ and $3 / 2$ components before the $3 / 2$ island grows up. In addition, the $2 / 2$ component amplitude is large only during the sawtooth collapse but much smaller afterwards, as shown in figures 15 and 16.

Again for the parameters of figure 15, the radial profiles of the normalized $m / n=0 / 0$ component plasma current density and electron density are shown in figure 17 (left and right) at $t=0,9.9 \times 10^{-5} \tau_{R}$ and $5.6 \times 10^{-4} \tau_{R}$. There are local perturbations in the $m / n=0 / 0$ component of the plasma current density and of the electron density around $r_{3 / 2}$ at $t=9.9 \times 10^{-5} \tau_{R}$, similar to what has been found in the oscillation regime of Section 3. The flattening of the $m / n=0 / 0$ component of the plasma current density and electron density in the central region is caused by the sawtooth crash. Later in time the 3/2 mode grows into the NTM regime. The electron density flattens around $r_{3 / 2}$, and the local $\mathrm{m} / \mathrm{m}=0 / 0$ component of the plasma current density perturbations disappear, similar to that of the NTM regime shown Section 3.
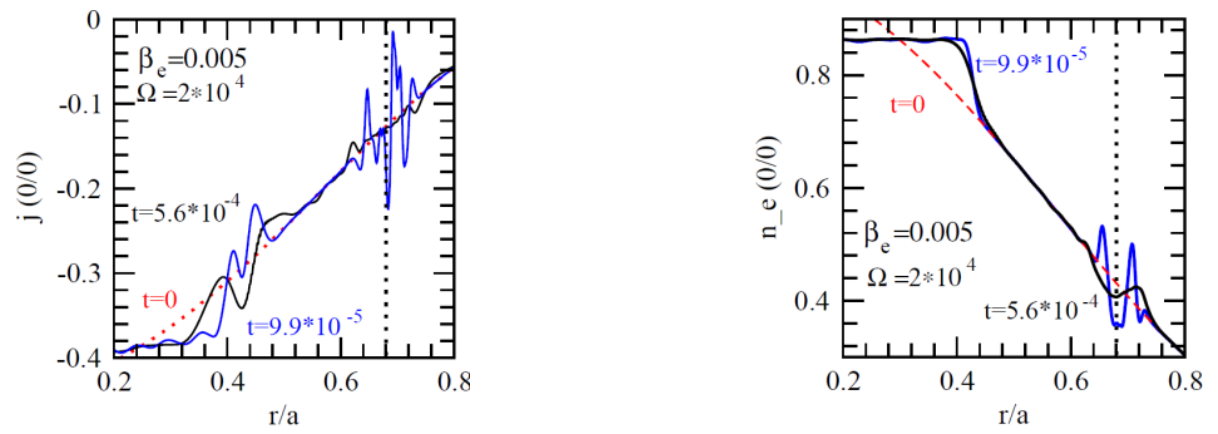

Figure 17 Corresponding to figure 15 , radial profiles of the normalized $m / n=0 / 0$ component of the plasma current density (left) and of the electron density (right) at $t=0,9.9 \times 10^{-5}$ and $5.6 \times 10^{-4} \tau_{R}$.

Increasing the value of $\Omega$ to $\Omega=4 \times 10^{4}$ while keeping other input parameters to be the same as those for figure 15 , the $3 / 2$ mode grows into the oscillation regime but not the NTM regime for $\beta_{e}=0.008$, indicating the stabilizing effect of a larger diamagnetic drift frequency. 
The time evolution of $W_{3 / 2} / \mathrm{a}$ is shown in figure 18 for different bootstrap current density. The black curve is the same as that in figure 16 (left), shown here for comparison. The red curve is for zero bootstrap current density, and the blue curve is obtained with half the bootstrap current density of that for the black curve, keeping other input parameters unchanged. The 3/2 mode grows into the NTM regime later for a lower bootstrap current density. Without the bootstrap current, the 3/2 mode only grows into the oscillation regime, showing the destabilizing effect of higher bootstrap current.

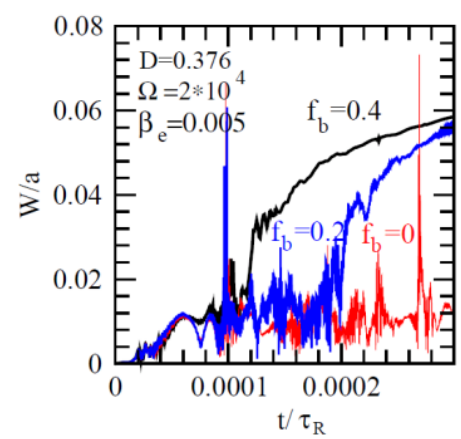

Figure 18 Time evolution of $W_{3 / 2} / a$. The black curve is the same as that in figure 16 (left), shown here for comparison. The red curve is for zero bootstrap current density, and the blue curve is obtained with half the bootstrap current density of that for the black curve, keeping other input parameters unchanged.

In the oscillation regime a high frequency mode with a frequency $f \sim 4 \times 10^{4} \mathrm{~Hz}$ is usually observed, and the shear Alfven frequency and the diamagnetic drift frequency are close at $\left|r-r_{3 / 2}\right| \sim 0.02 a$ similar to that shown in Section 3.

Increasing the perpendicular particle diffusivity by 10 times to $D_{\perp}=3.76 a^{2} / \tau_{R}$, the time evolution of $W_{3 / 2} / a$ (solid) is shown in Fig. 19 (left) for $\beta_{e}=0.006$ by the black curve, keeping the other input parameters the same as those for figure 15, The sawtooth crash happens at $t=2 \times 10^{-5} \tau_{R}$. Afterwards the 3/2 mode first grows into the oscillation regime and then into the NTM regime, similar to the case shown in figure 16. For the higher diffusion coefficient considered here, the oscillation regime lasts longer, until $t=0.00036 \tau_{R} \sim 8.3 \mathrm{~ms}$. After the $3 / 2$ mode enters the NTM regime, the $\psi_{3 / 2}$ profiles have the feature of a tearing mode. If the bootstrap current is taken to be zero, the $3 / 2$ mode only grows into the 
oscillation regime. Keeping other input parameters the same as those for the black, the $3 / 2$ mode remains at a very small amplitude for a lower value of $\beta_{e}, \beta_{e}=0.004$ (blue). When using an upwards shifted equilibrium q-profile with a smaller $r_{q=1}, r_{q=1}=0.18 a$, which results in a smaller sawtooth crash amplitude, while keeping other input parameters the same as those for the black curve, the $3 / 2$ mode only has a low amplitude (red).
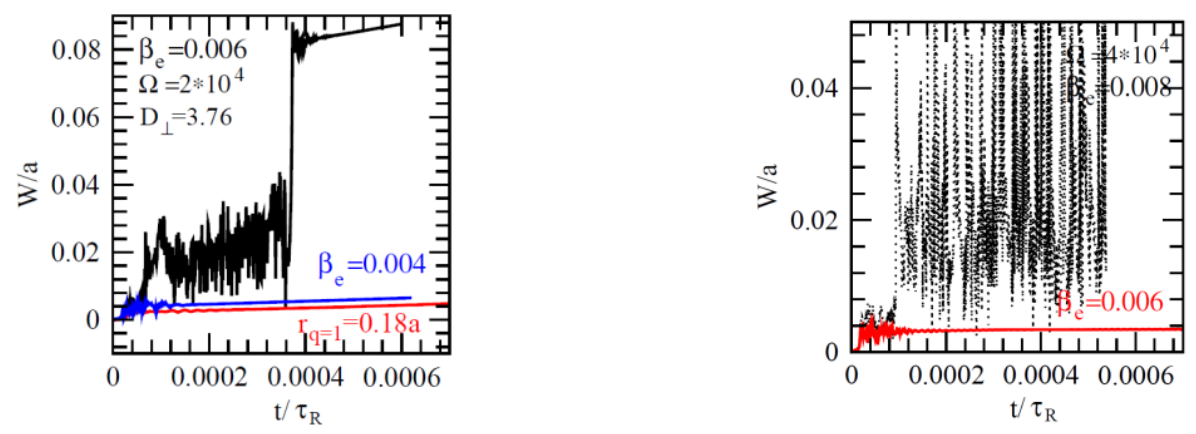

Figure 19 (left) Time evolution of $W_{3 / 2} / a$ for $D_{\perp}=3.76\left(a^{2} / \tau_{R}\right), \Omega=2 \times 10^{4}$ and $f_{b}=0.4$ at $r_{3 / 2}$. The black (blue) curve is obtained for $\beta_{e}=0.006$ (0.004). The red curve is obtained for an upwards shifted equilibrium q-profile $\left(r_{q=1}=0.18 a\right)$, keeping other input parameters the same as those for the black one. (right) Time evolution of $W_{3 / 2} / a$ with $\Omega=4 \times 10^{4}$ for $\beta_{e}=0.008$ (black curve) and 0.006 (red), keeping other input parameters the same as those for the black curve in the left figure.

For the case shown by the black curve in figure 19 (left), there is also a local perturbation in the $m / n=0 / 0$ component electron density around $r_{3 / 2}$ in the oscillation regime. After the $3 / 2$ mode enters into the NTM regime, the electron density flattens around $r_{3 / 2}$, similar to the results shown in figure 17 . The radial profiles of the normalized $m / n=0 / 0$ component plasma current density also have similar features to that shown figure 17 . In the oscillation regime a mode fluctuating around a frequency $f \sim$ $4 \times 10^{4} \mathrm{~Hz}$ is also seen due to the shear Alfven resonance.

Increasing the value of $\Omega$ by two times to $\Omega=4 \times 10^{4}\left(\Delta \omega *_{\mathrm{e}}=2.1 \times 10^{5} / \tau_{R}\right)$, the time evolution of $W_{3 / 2} / a$ is shown in Fig. 19 (right) for $\beta_{e}=0.006$ (red curve) and 0.008 (black). The other input parameters are the same as those for the black curve in figure 19 (left). The 3/2 mode saturates at a low level for 
$\beta_{e}=0.006$ but grows into the oscillation regime for $\beta_{e}=0.008$, showing again the destabilizing effect of higher $\beta_{e}$ value. However, the 3/2 mode has not grown into the NTM regime due to a larger $\Omega$ value. Comparing with figure 19 (left), it is found that a higher $\beta_{e}$ value is required to excite the $3 / 2$ mode for a larger value of $\Omega$.

The above results indicate that the triggering of a 3/2 magnetic island by a sawtooth crash is similar to the mode coupling to a $2 / 2$ tearing mode (without the $1 / 1$ mode involved). Both the oscillation and NTM regime shown in Section 3 are also found here. As the electron diamagnetic drift is stabilizing for the $3 / 2$ mode growth, while a high $\beta_{e}$ value enhances the mode coupling between the $2 / 2$ and $3 / 2$ components, the 3/2 mode is more easily triggered after the sawtooth collapse for a higher $\beta_{e}$ value and/or smaller electron diamagnetic drift frequency. The 3/2 mode saturates in the oscillation regime for a low bootstrap current density or a large diamagnetic drift frequency, but grows into the NTM regime in the opposite limit.

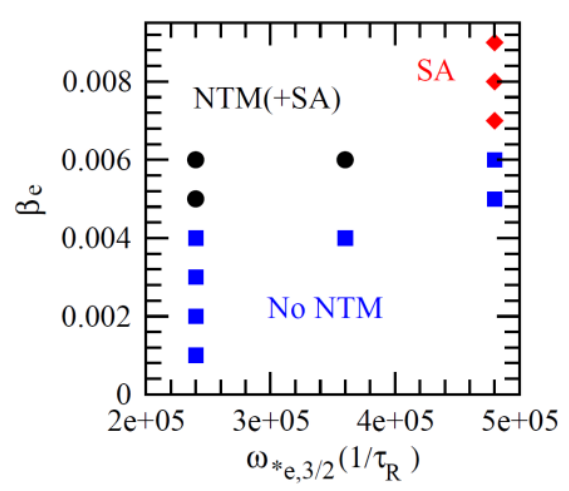

Figure 20 Stability diagram in the $\left(\omega_{*, 3 / 2}-\beta_{e}\right)$ plane for the destabilization of the 3/2 mode by sawteeth. The black circles correspond to the cases in which the 3/2 mode is destabilized first into the oscillation regime with shear Alfven resonance and then into NTM regime. The red diamonds correspond to cases in which the 3/2 mode is only destabilized into the oscillation regime. The blue squares correspond to the cases with no 3/2 mode destabilized. The required $\beta_{e}$ value for the 3/2 mode to enter the NTM regime significantly increases for a sufficiently large diamagnetic drift frequency at $r_{3 / 2}$. 
The results of this section are summarized in the $\left(\omega *_{e, 3 / 2}-\beta_{e}\right)$ plane in figure 20 . The other input parameters are the same as those for figure 15. The black circles correspond to the cases in which the 3/2 mode is destabilized first into the oscillation regime with shear Alfven resonance and then into the NTM regime. The red diamonds correspond to cases in which the $3 / 2$ mode is only destabilized into the oscillation regime. The blue squares correspond to the cases with no $3 / 2$ mode. The required $\beta_{e}$ value for destabilizing the $3 / 2$ mode significantly increases with increasing the local diamagnetic drift frequency at $r_{3 / 2}$. A larger perpendicular particle diffusivity results in a higher $\beta_{e}$ for destabilizing the 3/2 mode than that shown in figure 20.

\section{Discussion and summary}

The stabilizing role of the differential plasma rotation and the destabilizing role of a finite plasma $\beta$ value in mode coupling are found from the single fluid simulations, as expected from analytical theory [16-18]. The results obtained from the four-field equations are, however, much more complicated even without including the $1 / 1$ mode in the calculations. For a low electron diamagnetic drift frequency $\left(\left(\Omega \leq 2 \times 10^{4}\right.\right.$, corresponding to $\left.f_{*_{e}, 3 / 2} \leq 1.7 \mathrm{kHz}\right)$, the results are similar to that of the single fluid calculations. In this case, the 3/2 mode grows into the NTM regime for a quite low value of $\beta_{e}$ (=0.001). For a sufficiently large yet realistic value of the diamagnetic drift frequency $\left(\Omega \geq 4 \times 10^{4}\right)$, three regimes are found: The suppression regime exists for moderate $\beta_{e}$ values, in which the originally unstable $2 / 2$ mode is stabilized by its coupling to the $3 / 2$ mode, and the $3 / 2$ mode saturates at a low amplitude, as shown in Section 3.2.2. Increasing the value of $\beta_{e}$ to about 0.007 (depending on the value of the perpendicular particle diffusivity), an oscillation regime exists, in which the $3 / 2$ mode has the feature of an ideal mode. The NTM regime exists for a sufficiently large $\beta_{e}$ and bootstrap current density. The 3/2 mode first enters into the oscillation regime and then the NTM regime. If the bootstrap current density is too small, the 3/2 mode saturates in the oscillation regime. 
The simulation results of the destabilization of 3/2 modes by sawteeth are similar to those with a 2/2 tearing mode only (without the $1 / 1$ mode), but a higher $\beta_{e}$ value and a sufficiently large $r_{q=1}$ (or sawtooth amplitude) is required for the $3 / 2$ mode onset. The $3 / 2$ mode grows into the NTM regime only several $m s$ after the sawtooth crash, if the bootstrap current density is sufficiently large. These numerical results indicate that the electron diamagnetic drift, plasma beta value and the bootstrap current are important in determining the triggering of 3/2 tearing modes by sawteeth.

In the standard four-field equations, the electron temperature is assumed to be a constant in space and time [26]. As shown in Section 3.2.5, the term $T_{e} / n_{e}(r)$ in front of the parallel electron density gradient in Ohm's law affects the local electron diamagnetic frequency at $r_{3 / 2}$ for a given value of $\Omega$, and a higher value of $T_{e} / n_{e}(r)$ at $r_{3 / 2}$ corresponds to a larger local electron diamagnetic drift. As the local electron diamagnetic drift significantly affects the 3/2 mode onset, further extension of the standard four-field equations, to include the time evolution of the electron temperature, is required.

Analytical theories on shear Alfven resonance exist only for a single tearing mode in one-fluid model [58-61]. Further analytical works are required to include the mode coupling and two-fluid effects to compare with numerical results.

Experiments have been carried out in ASDEX upgrade, TCV and Tore Supra to study the triggering of NTMs by usual or monster sawteeth $[10,62,63]$. A quick growth of the $n=2$ magnetic perturbations right after a sawtooth is usually observed [10,62], as also seen in our simulations. Sawteeth with longer period, usually corresponding to larger amplitude, are found to more easily generate NTMs [62]. This is particularly true for the $3 / 2$ NTMs with significant $(2,2)$ component triggered by monster sawteeth, which have a large minor radius of $\mathrm{q}=1$ surface [63]. After sawtooth crash the magnetic island is measured a few ms later or even longer on ASDEX upgrade [10]. About the same time scale is seen from our numerical results. As pointed out in Ref. [10], it is possible to identify the mode type (ideal or tearing) in experiments after the crash only with local measurements, e.g. ECE diagnostic, and the 
island can be measured only when it is sufficiently large ( $1 \mathrm{~cm}$, depending on measurement accuracy). All measurements related to the mode structure identification in Ref. [62] were however non-local, made either outside the plasma (magnetic probes) or using line integrated signs (Soft X-ray cameras).

We would like to emphasize again the limitations of our studies. Our results are based on the fourfield equations that neglect higher order terms in large aspect ratio expansion, which is of particular importance for the 1/1 internal kink mode. The cold ion assumption has been made. Sawtooth amplitude is determined only by taking different equilibrium q-profiles. Our results are affected by the value of, e.g. the Lundquist number and the parameter $d_{1}$ in the electron continuity equation, which are known to be related the ion polarization current [35], suggesting its role in the NTM stability. The ion polarization current is self-consistently described by the four-field equations only in the fluid or collisional regime [26,35,36], while kinetic corrections in the collisionless regime, as described in Ref. [36], have not been included, which would require a modification of the equations in the future.

In summary, numerical simulations have been carried out to understand the mode coupling and seeding of NTMs. Within single fluid model the mode coupling is found to be weakened by relative mode rotation but enhanced by finite plasma $\beta$ value. Using the four-field equations, the 3/2 NTMs are found to be triggered by sufficiently strong sawtooth crashes or $2 / 2$ perturbations at higher $\beta$ value and bootstrap current density and/or a lower electron diamagnetic drift frequency. Before the 3/2 mode grows into the NTM regime, an oscillation regime is found existing for a sufficiently large diamagnetic drift frequency and $\beta$ value, in which the diamagnetic drift frequency reaches the shear Alfven frequency outside the tearing layer and causes shear Alfven resonance. 


\section{References}

[1] Chang Z. et al 1995 Phys. Rev. Lett. 744663

[2] Zohm H. et al 2001 Phys. Plasmas 82009

[3] Günter S. et al 2001 Phys. Rev. Lett. 87275001

[4] La Haye R.J., Lao L.L., Strait E.J. and Taylor T.S. 1997 Nucl. Fusion 37397

[5] Sauter O. et al 1997 Phys. Plasmas 41654

[6] Gude A., Günter S. and Sesnic S. et al 1999 Nucl. Fusion 39127

[7] La Haye R. J. 2006 Phys. Plasmas 13055501

[8] Fredrickson E.D. 2002 Phys. Plasmas 9548

[9] Buttery R.J. et al 2008 Phys. Plasmas 15056115

[10] Igochine V. et al 2014 Phys. Plasmas 21110702

[11] Igochine V. et al 2017 Nucl. Fusion 57116027

[12] Wesson J. A. 1978 Nucl. Fusion 18, 87

[13] Wesson J. A., Gill R. D., Hugon M. et al 1989 Nucl. Fusion 29, 641

[14] Trier E. et al 2019 Plasma Phys. Control. Fusion 61, 045003

[15] Connor J W et al 1988 Phys. Fluids 31577

[16] Cowley S C and Hastie R J 1988 Phys. Fluids 31426

[17] T. C. Hender et al 1992 Nucl. Fusion 32, 2091

[18] Fitzpatrick R. 1994 Phys. Plasmas 13308

[19] Carreras B., Waddell B.V., and Hicks H. R. 1979 Nucl. Fusion 19, 1423

[20] Ham C J et al 2012 Plasma Phys. Control. Fusion 54025009

[21] Lütjens H, Luciani J-F and Garbet X 2001 Phys. Plasmas 84267

[22] Lütjens H, Luciani J-F and Garbet X 2001 Plasma Phys. Control. Fusion 43 A339

[23] Sugiyama L.E. 2015 Nucl. Fusion 55073006 
[24] Sugiyama L.E. 2013 Phys. Plasmas 20032504

[25] Sugiyama L.E. 2014 Phys. Plasmas 21022510

[26] Hazeltine R. D. et al 1985 Phys Fluids 282466

[27] Strauss H. R. 1977 Phys. Fluids 201354

[28] Strauss H. R. 1981 Phys. Fluids 24, 2004

[29] Glasser A. H., Greene J. M. and Johnson J. L. 1975 Phys. Fluids 18875

[30] Glasser A. H., Greene J. M. and Johnson J. L. 1976 Phys. Fluids 19567

[31] Grimm R. C., Greene J.M. and Johnson J. L. Methods in Computational Physics, edited by Killeen

J. (Academic Press, New York, 1976), Vol. 16, p. 253

[32] Strumberger E. and Günter S. 2017 Nucl. Fusion 57016032

[33] Orain F., Hölzl M., Viezzer E. et al 2017 Nucl. Fusion 57022013

[34] Orain F., Bécoulet M., Morales J. et al 2013 Plasma Phys. Controlled Fusion 55014020

[35] Smolyakov A.I. 1993 Plasma Phys. Control. Fusion 35657

[36] Wilson H.R., Connor J.W., Hastie R.J. and Hegna C.C. 1996 Phys. Plasmas 3248

[37] Connor J.W., Waelbroeck F.L. and Wilson H.R. 2001 Phys. Plasmas 82835

[38] Waelbroeck F.L., Connor J.W. and Wilson H.R. 2001 Phys. Rev. Lett. 87215003

[39] Fitzpatrick R., Waelbroeck F.L. and Militello F. 2006 Phys. Plasmas 13122507

[40] Waelbroeck F.L. and Fitzpatrick R. 1997 Phys. Rev. Lett. 781703

[41] Drake J.F., Antonsen T.M., Hassam A.B. and Gladd N.T., 1983 Phys. Fluids 26, 2509

[42] Scott B.D. and Hassam A.B., 1987 Phys. Fluids 30, 90

[43] Yu Q. 2010 Nucl. Fusion 50025014

[44] Halpern F.D., Lütjens H. and Luciani J-F, 2011 Phys. Plasmas 18, 102501

[45] Halpern F.D., Leblond D., Lütjens H. and Luciani J-F, 2011 Plasma Phys. Control. Fusion, 53, 015001

[46] Ahn J.-H., Garbet X., Lütjens H. et al, 2016 Phys. Plasmas 23, 052509 
[47] Yu Q., Günter S. and Lackner K. 2015 Nucl. Fusion 55113008

[48] Günter S., Yu Q., Lackner K., Bhattacharjee A., Huang Y-M, 2015 Plasma Phys. Control. Fusion, 57, 014017

[49] Beidler M.T., Cassak P.A., S C Jardin S.C. and Ferraro N. M., 2017 Plasma Phys. Control. Fusion, 59, 025007

[50] Brennan D.P., Kruger S.E., Gianakon T.A. and Schnack D.D. 2005 Nucl. Fusion 451178

[51] Popov A.M., La Haye R. J., Liu Y.Q. et al, 2002 Phys. Plasmas 94205

[52] Brennan D.P. and L. E. Sugiyama L.E., 2016 Phys. Plasmas 13, 052515

[53] O. Février T. Nicolas T., Maget P. et al 2018 Nucl. Fusion 58096008

[54] Brunetti D., Graves J.P., Halpern F.D. et al 2015 Plasma Phys. Control. Fusion 57054002

[55] Kleiner A., Graves J.P., Brunetti D., et al 2016 Nucl. Fusion 56092007

[56] Yu Q. and Günter S. 2011 Nucl. Fusion 51073030

[57] Yu Q., Günter S. and Lackner K. 2018 Nucl. Fusion 58054003

[58] Wang X., Bhattacharjee A., Ma Z. W., Ren C., Hegna C. C., and Callen J. D. 1998 Phys. Plasmas 5, 2291

[59] Liu Y., Connor J. W., Cowley S. C., Ham C. J., Hastie R. J., and Hender T. C. 2012 Phys. Plasmas 19102507

[60] Wei L. and Wang Z. 2013 Plasma Phys. Controlled Fusion 55085004

[61] Hu Q., Yu Q. and Hu X. 2014 Phys. Plasmas 21122507

[62] Canal G.P., Duval B. P., Felici F. et al 2013 Nucl. Fusion 53113026

[63] Maget P. et al, 2005 Plasma Phys. Control. Fusion 47357 